\section{Aircraft system-level diagnosis with emphasis on maintenance decisions}

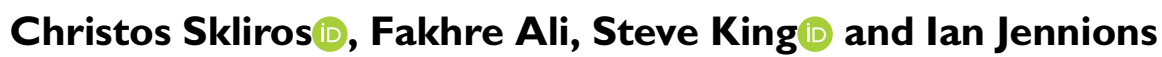

Proc IMechE Part O:

$\mathrm{J}$ Risk and Reliability

2022, Vol. 236(6) 1057-1077

(c) IMechE 2021

(c) (i) (5)

Article reuse guidelines:

sagepub.com/journals-permissions DOI: $10.1177 / 1748006 \times 211054938$ journals.sagepub.com/home/pio

@SAGE

\begin{abstract}
This paper proposes a diagnostic technique that can predict component degradation for a number of complex systems. It improves and clarifies the capabilities of a previously proposed diagnostic approach, by identifying the degradation severity of the examined components, and uses a 3D Principal Component Analysis approach to provide an explanation for the observed diagnostic accuracy. The diagnostic results are then used, in a systematic way, to influence maintenance decisions. Having been developed for the Auxiliary Power Unit (APU), the flexibility and power of the diagnostic methodology is shown by applying it to a completely new system, the Environmental Control System (ECS). A major conclusion of this work is that the proposed diagnostic approach is able to correctly predict the health state of two aircraft systems, and potentially many more, even in cases where different fault combinations result in similar fault patterns. Based on the engineering simulation approach verified here, a diagnostic methodology suitable from aircraft conception to retirement is proposed.
\end{abstract}

\title{
Keywords
}

Aeronautical asset management, aeronautical maintenance, aerospace maintenance, condition based maintenance, failure detection, fault diagnostics, fault isolation, fault monitoring

Date received: 9 May 2021; accepted: 26 September 2021

\section{Introduction}

According to the analysis by Saxon and Weber, ${ }^{1}$ maintenance costs contribute significantly to the overall operating cost of an airline, and as the International Air Transport Association (IATA) ${ }^{2}$ reports, Maintenance Repair and Overhaul (MRO) represents a $\$ 76 \mathrm{~B} /$ year market. Accurate identification of system and component health states enable optimization of an airline's maintenance plan, which finally results in increased asset availability and reduced cost. ${ }^{3}$ Therefore, diagnostic techniques, that can correctly identify the system and component health states, are helpful for airlines and maintenance organizations. As the major drivers of aircraft maintenance are the Auxiliary Power Unit (APU) ${ }^{4}$ and the Environmental Control System $(\mathrm{ECS})^{5}$ these systems are analyzed in this paper.

This paper proposes a diagnostic technique that can predict component degradation for any complex system. It builds on previous work on detecting APU faults in the presence of other degraded components. The development of the diagnostic methods in this work considers the fact that degradation builds-up in all system components from the moment they are installed on the aircraft, and consequently all components can be subject to a certain degree of degradation. Therefore, the most critical challenge from a maintainer's point of view is the correct identification of each component's degradation level. This is in order to promptly replace components that are approaching the end of their lives, while avoiding unnecessary replacement of components with minimal degradation (overmaintenance).

The aim of this work is to design a diagnostic methodology that is able to identify the degradation level of the examined components and correlate them with appropriate maintenance action. In order to achieve this, a traffic light-based system is adopted to define three different fault severity regions, each one of them associated with a different maintenance action.

Integrated Vehicle Health Management Centre, School of Aerospace, Transport and Manufacturing, Cranfield University, Bedfordshire, UK

\section{Corresponding author:}

Christos Skliros, Integrated Vehicle Health Management Centre, School of Aerospace, Transport and Manufacturing, Cranfield University, College Road, Bedfordshire MK43 0AL, UK.

Email: C.Skliros@cranfield.ac.uk 
Following that, two different diagnostic approaches are compared on the APU system in order to establish a robust methodology. The approach that demonstrated the best performance on the APU case study is applied on a new system, in this case the ECS.

Finally, challenges that can be encountered when applying the proposed approach in "real-world" conditions are discussed and a diagnostic methodology suitable for use with an aircraft throughout its life cycle is proposed. The most prominent implementation issues addressed are:

- In some cases only limited data is available to train and test the diagnostic algorithm. For this reason, the proposed technique was evaluated with sparse datasets.

- The definition of the thresholds of the different severity levels heavily depends on field data. Therefore, the proposed fault severity regions that are proposed throughout this paper are based on data from the public domain literature and the authors' experience. End-users with access to the relevant field data can define their own thresholds and associate them with the relevant maintenance actions.

Based on the success of the proposed diagnostic approach on two different systems, the main characteristics of this methodology are generalized and, by considering the implementation challenges, a through-life diagnostic approach to maintenance is proposed. This approach, which is based on the Condition-Based Maintenance (CBM) philosophy, can be applied to any complex system that consists of multiple sub-systems with replaceable components including control loops and is structured to serve both new and legacy designs. The proposed through-life diagnostic approach constantly re-defines the thresholds of the severity regions based on field data in order to create the most suitable thresholds specifically tailored for each system.

\section{Background of APU diagnostics}

The APU is a system installed in all commercial aircraft and its purpose is to provide bleed air and electric power to other aircraft systems. Skliros et al. ${ }^{6}$ describe the principle of operation of a Boeing 747 APU and discuss its performance characteristics under various operating conditions. The APU belongs to the broader category of Gas Turbine (GT) engines, for which a large variety of diagnostic techniques have been developed. $\mathrm{Li}^{7}$ and Tahan et al. ${ }^{8}$ present the most important model-based diagnostic approaches for GT systems, and Bettocchi et al. ${ }^{9,10}$ discuss the most representative data-driven diagnostic techniques.

Since the APU is a major contributor to aircraft maintenance, a number of research studies discuss diagnostic approaches specifically for APU systems. Some of the most representative approaches are mentioned below. Gorinevsky et al. ${ }^{11}$ developed a model-based methodology to diagnose faults in APU components during start-up and steady state operation. Also, Vianna et al. ${ }^{12}$ used a classification tree to diagnose excessive bleed, inlet blockage and fuel filter blockage faults of an APU, by monitoring field data (Exhaust Gas Temperature (EGT), bleed pressure, and fuel flow). Pascoal et al. ${ }^{13}$ trained neural networks to predict the changes in basic APU performance parameters (bleed mass flow, compressor efficiency, turbine efficiency), based on data produced by a simulation model. Finally, in the work conducted by Guralnik et al. ${ }^{14}$ the probabilities of various APU faults were calculated by integrating the results of component-level diagnostic algorithms based on the Dempster-Shafer fusion rules.

A full literature review in APU and GT health monitoring methods ${ }^{15}$ shows that most diagnostic studies consider only the GT sub-system, while the ancillary sub-systems (e.g. fuel system, control system, and electric system) are ignored. However, as has been discussed by Skliros et al. ${ }^{15}$ faults in the ancillary subsystems can result in global changes in the system's performance, and for this reason it is important to consider all sub-systems when conducting health monitoring studies.

\section{Background of ECS diagnostics}

The ECS is a system installed in all commercial aircraft with the purpose of providing conditioned air to the passenger cabin, by processing bleed air (from the engines or the APU) through a series of mechanical components. The principle of operation of a Boeing 737-800 ECS is presented in the work conducted by Jennions et al. ${ }^{16}$ in which a simulation model for the ECS is developed and its performance characteristics are explored for various boundary conditions.

Most ECS diagnostic studies discuss single component faults, and diagnostic studies for heat exchanger fouling dominate the public domain literature. A diagnostic study for heat exchanger fouling has been conducted by Najjar et al. ${ }^{17}$ In this approach, a physicsbased model was used to generate the necessary data, and based on the most relevant sensor suite, machine learning algorithms were trained to diagnose heat exchanger fouling. Also, Shang and $\mathrm{Liu}^{18}$ proposed a technique that is able to diagnose heat exchanger fouling by monitoring the changes in the Temperature Control Valve's (TCV) position. Finally, Shah et al. ${ }^{19}$ using a state space model of the heat exchanger were able to predict changes in the performance characteristics via the Extended Kalman Filter (EKF).

However, as has been discussed by Jennions, ${ }^{20}$ the ECS control system masks the effects of component degradation, and hence the correct identification of the system health state requires system-level, rather than component-level analysis. Despite the fact that systemlevel analyses are necessary for the accurate identification of the system health state, they are rarely available 
in the public domain literature. Najjar et al. ${ }^{17}$ discuss heat exchanger fouling faults by taking into account the operation of the entire ECS system. An important observation in the study by Najjar et al. ${ }^{17}$ is the fact that degradation is considered only in the heat exchanger, while all other components are assumed to be free from degradation. However, as will be discussed in the rest of this paper, in order to accurately diagnose the system health state, and proceed to corrective actions, simultaneous existence of degradation in all components should be considered.

As regards system-level diagnostic studies that consider the simultaneous existence of multiple faults, Liu et al. ${ }^{21}$ developed a diagnostic technique that is able to diagnose multiple faults in an ECS. This is done by a Bayesian network, which is constructed based on first principles, expert knowledge, and maintenance data and is designed to detect faults in the following components: heat exchanger, air cycle machine, temperature control valve and ram air actuator. Finally, Hare et al. ${ }^{22}$ trained multiple neural networks in order to differentiate between heat exchanger, air cycle machine, and sensor faults. An interesting aspect of their approach is that a dedicated neural network was trained to recognize the health state of each examined component. Furthermore, each neural network was trained to recognize the healthy state of its corresponding component, while another component can be degraded. This training methodology significantly increased the accuracy of the diagnostic results when compared with the classical training approach, that under the healthy state, all components are healthy. The training methodology used in the work by Hare et $\mathrm{al}^{22}$ has motivated the diagnostic approach presented in this paper.

\section{Scope of the present work}

The main observation coming out of the literature survey on APU, GT, and ECS diagnostic studies, is the fact that most diagnostic approaches are based on the "single fault" hypothesis, which means that the diagnostic analyses assume that only one component can be degraded. However, in real systems, all components start to degrade from the moment the system is installed on the aircraft, and consequently degradation is to be expected in all components simultaneously. For this reason, in order to accurately assess the system health state, the diagnostic algorithms should consider the simultaneous existence of multiple component degradation, at different severity levels.

Even though the simultaneous existence of multiple faults is frequently observed in industry, the relevant literature is limited. The most important diagnostic studies discussing the simultaneous existence of multiple faults (relevant to the systems examined in this paper) have been applied mostly on GT engines while only a few papers consider multiple faults in ECS systems. A representative study discussing multiple fault diagnostics for a GT system has been conducted by Sadough Vanini et al. ${ }^{23}$ and in this work, the researchers tested the ability of a parallel bank of autoassociative neural networks to detect the simultaneous existence of component and sensor faults. The results showed that the proposed approach is able to detect the simultaneous existence of a sensor and a component fault, however, this technique was not tested for simultaneous faults in different components. Joly et al. ${ }^{24}$ proposed a technique that can diagnose five single component faults and three double component faults in a turbofan engine by training a neural network for each fault combination that is examined. The proposed technique was able to correctly predict the single and double faults tested, however a disadvantage of this technique is that the responses of the diagnostic algorithms are limited to the fault combinations considered during training. Therefore, in order to identify all possible fault combinations, that can appear in a real engine, the classifier must consider many target classes, and this might reduce its ability to define accurate decision boundaries. Lee et al. ${ }^{25}$ developed a technique that is able to diagnose sensor and component faults (both single and multiple) using a hierarchical Bayesian network. The proposed approach was able to predict a compressor fault and a fuel flow sensor bias, however, this technique should be further tested under multiple faults. Furthermore, Sampath and Singh ${ }^{26}$ have proposed a technique that combines neural networks with genetic algorithms to diagnose multiple faults in a GT. This approach leveraged both the high accuracy offered by the genetic algorithms and the fast response of neural networks. The proposed technique was able to successfully diagnose a sensor fault as well as single and multiple component faults (in no more than two components). However, the classification approach used in this paper would require an excessive number of target classes in order to diagnose the simultaneous existence of sensor and component faults (more than two), which could impact the predictions' accuracy. Finally, Guralnik et al. ${ }^{14}$ used Dempster-Shafer (D-S) theory to integrate the results of independent singlefault diagnostic algorithms in order to calculate the probability of simultaneous multiple faults in an APU system.

As regards the ECS, the most notable studies discussing multiple faults include Hare et al. ${ }^{22}$ who used neural networks to identify the simultaneous existence of heat exchanger, air cycle machine, and sensor faults. Also, as described previously, Liu et al. ${ }^{21}$ developed a Bayesian network capable of diagnose simultaneous faults in four components. Finally, Palmer et al. ${ }^{27}$ developed a technique that is able to diagnose air cycle machine degradation, pipe corrosion, and degradation in the temperature control valve. Under the latter approach, a state space model of the ECS was developed and the target faults are diagnosed by analyzing the residual of the system outputs between the healthy and faulty conditions. Finally, diagnostic studies based 
on multi-component degradation have also been applied in other systems such as the fuel system. The most representative study for the fuel system has been conducted by Lin et al. ${ }^{28}$ in which a Bayesian network was used to identify the component degradation severity.

The current authors, in their previous work, ${ }^{15}$ conducted a diagnostic analysis for a Boeing 747 APU that considered simultaneous faults in the Compressor, Turbine, Load Control Valve (LCV), Fuel Metering Valve (FMV), and Electric Generator. The healthy and faulty data were generated by a simulation model that was calibrated against experimental data. By assessing the model's sensitivity for various fault cases, it was seen that different fault combinations result in similar fault patterns. Therefore, for each component under examination, an individual classification algorithm was trained to recognize its healthy and faulty state, while other components could be either healthy or faulty. The most important element in the design of the diagnostic classifiers relied on the fact that for each examined component four different scenarios were considered to label the datasets that corresponded to the healthy and the faulty cases. For example, the data that corresponded to the healthy case were generated by simulations in which the examined component was always healthy, while the other components were subject to various degradation levels. The same methodology was adopted to generate the datasets that correspond to the faulty case. Finally, when evaluated against test datasets, the proposed diagnostic technique was able to predict component faults (without considering fault severity levels), even for test cases that caused similar changes to the system's performance. Based on the aforementioned background, the aim of this work is to clarify and improve the capabilities of the diagnostic approach previously proposed, ${ }^{15}$ by identifying the degradation severity of the examined components, and discuss how diagnostic results influence maintenance decisions. In the rest of this paper the methodology that will be used to generate training and testing datasets as well as the design and structure if the diagnostic classifiers will be thoroughly discussed.

Furthermore, the current work postulates that the proposed diagnostic methodology can be applied to any complex system, and for this reason, the case studies for the APU and the ECS are considered. The objectives of this work are:

- To enhance the authors previous work on APU diagnostics ${ }^{15}$ by:

- creating a diagnostic framework that is able to diagnose the degradation severity level of the examined component.

- comparing the performance of two different diagnostic approaches.

- discussing the implementation challenges of the proposed techniques in "real world" applications.
- To take the diagnostic approach with the best performance in the APU case studies and apply it (without modification) to a new system, the ECS.

- Given a successful outcome to the above, propose a diagnostic methodology that could live with the aircraft, supporting maintenance activity, through its life.

At this point it should be mentioned that, even though this work is motivated by the authors' previous work, ${ }^{15}$ the methods used to carry out the diagnostic analysis are thoroughly explained in this paper and consequently this paper is a self-contained piece of work. Namely, the fault modes considered in this analysis are detailed in Section 2, while in Section 3 all aspects of the diagnostic analysis are explicitly discussed. Especially as regards the diagnostic analysis, the objectives of the analysis are clearly stated and a comparison of two different approaches is conducted. For each diagnostic approach, the training, validation, and testing strategy are detailed, and the conclusions of this comparative study are discussed. Thereafter, based on the finding of the APU diagnostic analysis as presented in Section 3, the same methodology is applied on the ECS, in order to evaluate the ability of the proposed methodology to successfully detect the health state of a different system (Section 4). Finally, considering the outputs of the APU and ECS diagnostic analysis, Section 5 suggests a generic framework for Condition-Based Maintenance for aircraft systems.

In conclusion, it should be highlighted that the design of the overall diagnostic framework aims to optimize maintenance actions and this association is discussed throughout the paper. The importance of associating the diagnostic outputs with maintenance decisions is necessary since component replacement depends on their degradation level. In general, components should be replaced when they suffer from high severity degradation, in order to avoid system faults, while they should remain in the system when they are subject to low severity degradation. Therefore, the identification of the component degradation level determines the relevant maintenance decisions, which in turn modifies the overall maintenance plan of an organization.

\section{Fault simulations and diagnostic framework}

The necessary data to conduct the APU and the ECS diagnostic analyses are generated by models that are able to simulate these systems under healthy and faulty conditions. In this section, the fault simulation methodology is detailed, and the overall diagnostic framework is defined. As regards the faults considered in this work, these are selected based on the most typical fault modes mentioned in the public domain literature. Initially, the APU fault simulations are discussed, and 
Table I. Compressor and turbine degradation severity regions.

\begin{tabular}{llll}
\hline Component & Low severity & Medium severity & High severity \\
\hline Compressor & Mass flow decrease: $0 \%-1 \%$ & Mass flow decrease: $1.01 \%-4 \%$ & Mass flow decrease: $4.01 \%-5 \%$ \\
& Efficiency decrease: $0 \%-0.5 \%$ & Efficiency decrease: $0.505 \%-2 \%$ & Efficiency decrease: $2.005 \%-2.5 \%$ \\
Turbine & Mass flow decrease: $0 \%-1 \%$ & Mass flow decrease: $1.01 \%-4 \%$ & Mass flow decrease: $4.01 \%-5 \%$ \\
& Efficiency decrease: $0 \%-0.5 \%$ & Efficiency decrease: $0.505 \%-2 \%$ & Efficiency decrease: $2.05 \%-2.5 \%$ \\
\hline
\end{tabular}

Table 2. LCV fault classes.

\begin{tabular}{lll}
\hline Component & Valve healthy & Valve blocked \\
\hline LCV & Mass flow decrease: $0 \%$ & Mass flow decrease: $0.01 \%-5 \%$ \\
\hline
\end{tabular}

following that, the ECS fault simulations are presented. The diagnostic framework is designed in order to inform the operators of the most appropriate maintenance actions, based on the component degradation severity level. The diagnostic analysis in this paper is based on the hypothesis that all components start to degrade from the moment the system starts operation, and for this reason, low severity degradation should be acceptable. Furthermore, the outputs of the diagnostic analysis are defined in such a way, in order to inform the operators whether maintenance should be carried out immediately, or components should be replaced during the next available maintenance slot.

On this basis a traffic-light taxonomy system can be defined, that associates component fault severity with a maintenance action, as:

- Low Severity (Green): No maintenance action is necessary.

- Medium Severity (Amber): A maintenance action (for the corresponding component) should be carried out the next time the aircraft is grounded for maintenance for any scheduled or unscheduled inspection. It is not usually required to ground the aircraft when medium severity degradation is detected.

- High Severity (Red): High risk of system malfunction. The system should be shut down and maintenance should be carried out immediately.

Levels for these categories are given below and discussed, for the APU and the ECS respectively.

\section{APU component faults}

Tables 1 and 2 present the fault severity classes for the APU components (compressor, turbine, and LCV). In the rest of this section the methodology followed to select these fault modes and the threshold selection between the different fault classes is detailed.

APU performance data, that are used in this paper, are generated by the Boeing 747 APU simulation model discussed in Skliros et al. ${ }^{15}$ Figure 1 presents the schematic diagram of the APU model, along with its inputs and outputs. The model's output, which may be considered to come from virtual sensors, constitute the symptom vector, that will be used as an input in the diagnostic algorithms. For the purposes of this analysis, the simulated data correspond to ambient temperature $293^{\circ} \mathrm{K}$, while the APU is stationary at sea level. The bleed mass flow is defined as fraction of the inlet mass flow, and for this case study the bleed mass flow is $28 \%$ of the inlet mass flow (for the given environmental conditions this equals to $3 \mathrm{~kg} / \mathrm{s}$ ). Finally, the electric power produced by the electric generator is $25 \mathrm{~kW}$.

The diagnostic analysis conducted in Skliros et al. ${ }^{15}$ revealed that faults in the Compressor, Turbine, and Load Control Valve (LCV) always cause similar changes to the system's performance and for this reason, these components will be further examined in this analysis. Each of the examined components can develop a number of different fault modes depending on the changes in their characteristics. In order to identify the failure mechanism for each fault and their typical severity levels, information from the public domain literature is sought.

As regards the compressor, deformation of the surface and geometry of its blades change this component's characteristics (mass flow rate, pressure ratio, and isentropic efficiency). As has been reported by Kurz and Brun, ${ }^{29}$ a compressor fault typical changes all component characteristics, however, depending on the nature of the fault mode, some characteristics might have a stronger change compared to the others. For example, increased tip clearance has been reported by Graf et al. ${ }^{30}$ to strongly affect all compressor characteristics, while fouling or erosion faults result in a much stronger reduction in the mass flow and isentropic efficiency compared to the pressure ratio, as reported by Igie et al. ${ }^{31}$ and Zwebek and Pilidis. ${ }^{32}$ An area that requires further research is a more precise correlation between the changes in the component characteristics and the fault severity level, since very limited work has 


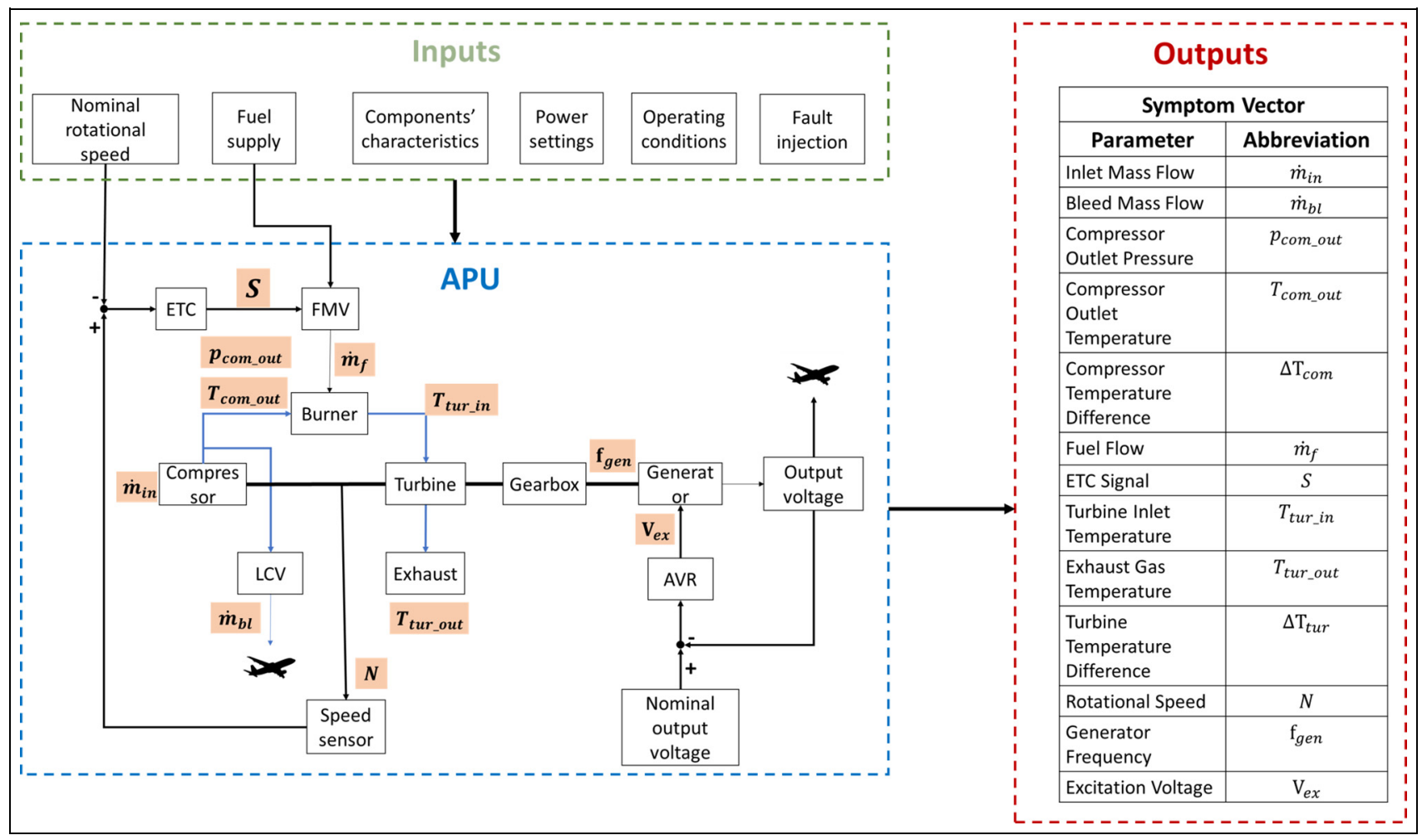

Figure I. APU simulation model schematic. ${ }^{15}$

been published on this issue. For the purposes of this work, the fault considered in the compressor emulates a fouling condition as described by Zwebek and Pilidis. ${ }^{32}$ The fault simulations in this paper consider reduction in the mass flow rate (up to $5 \%$ ) and decrease in the isentropic efficiency (up to $2.5 \%$ ), while the pressure ratio characteristic remains unaffected. The simulations carried out consider 500 partitions of the mass flow and efficiency with proportional changes for these characteristics. So, for example, if the mass flow rate is reduced by $1 \%$, this corresponds to a reduction in efficiency by $0.5 \%$.

As regards the turbine, similarly to the compressor, faults in this component are generated from changes in its blades surface and geometry. A turbine fault normally affects all turbine characteristics (mass flow rate and efficiency). However, depending on the existing fault mode, one of the turbine characteristics can have a much stronger influence than the other. For example, Boyle $^{33}$ and Kurz et al. ${ }^{34}$ report that the surface roughness and fouling cause a much stronger reduction in efficiency compared to the mass flow rate. In this paper, there is emulated a turbine fouling condition as reported by Zwebek and Pilidis. ${ }^{32}$ This condition results in a decrease in both the mass flow capacity (up to $5 \%$ ) and the efficiency (up to $2.5 \%$ ). Also, similarly with the compressor, it is assumed that the turbine's mass flow rate characteristic and the efficiency characteristic present linear changes to each other and with the same number of partitions, as in the compressor case.
Finally, the LCV is a pneumatically operated valve and as reported by Shang and $\mathrm{Liu}^{35}$ and Daigle and Goebel, ${ }^{36}$ the most typical fault mode developed in this component is a malfunction of the valve's actuator, due to degraded springs or excessive piston friction. Under the influence of these faults, the valve cannot be controlled properly and consequently this results in deviations of the provided bleed mass flow compared to the operator's commands. For the purposes of this application, the LCV fault mode emulates a condition that the valve is stuck and provides less bleed flow compared to the operator's command.

As mentioned in the Section 1, the aim of this work is to assess the ability of the proposed diagnostic approaches to identify the health state of the examined components. In order to compare the different diagnostic approaches, the emulated component faults should create similar changes on the system's performance. Thus, by taking into account the compressor and turbine faults, the LCV fault emulates a valve blockage that results in a reduction in the bleed mass flow up to $5 \%$ from its nominal value, which is a similar with the changes caused by the compressor and turbine degradation. Based on the fault modes defined above, the challenge to correctly diagnose the health state of the APU components can be seen by observing Figure 2 . In this figure, it is seen that three different fault combinations result in similar changes on the system's performance. Thus, the diagnostic algorithms should be able to identify each component's degradation 


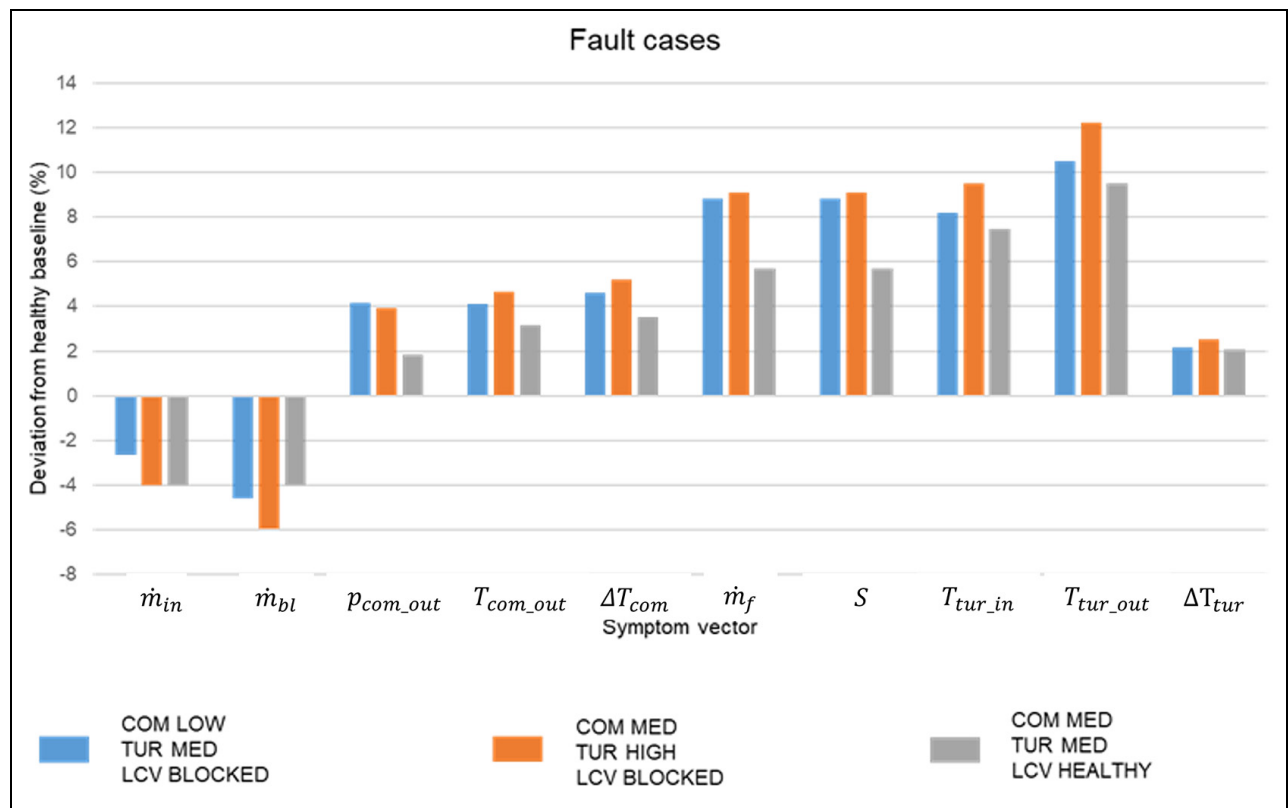

Figure 2. Examples of different APU fault cases that have similar fault patterns.

Table 3. ECS degradation severity regions.

\begin{tabular}{llll}
\hline Component & Low severity & Medium severity & High severity \\
\hline Primary heat exchanger & Efficiency decrease: $0 \%-25 \%$ & Efficiency decrease: $25 \%-50 \%$ & Efficiency decrease: $50 \%-75 \%$ \\
$\begin{array}{l}\text { Secondary heat exchanger } \\
\begin{array}{l}\text { Air cycle machine (turbine } \\
\text { degradation) }\end{array}\end{array}$ & Efficiency decrease: $0 \%-25 \%$ & Efficiency decrease: $25 \%-50 \%$ & Efficiency decrease: $50 \%-75 \%$ \\
\hline
\end{tabular}

characteristics and define decision boundaries that can accurately separate the different target classes.

\section{ECS component faults}

Table 3 shows the fault severity classes for the ECS components (Primary Heat Exchanger (PHX), Secondary Heat Exchanger (SHX), and Air Cycle Machine - Turbine (ACM-TUR)). In a similar way to the APU above, the methodology followed to select these fault modes and the threshold selection between the different fault classes is now detailed.

Performance data for the ECS is generated from a physics-based model that is able to simulate a Boeing 737-800 ECS under healthy and faulty conditions. This model has been developed by Jennions et al. ${ }^{16}$ and its sensitivity under component faults has been assessed by Jennions. ${ }^{20}$ Similarly to the APU, the ECS model calculates the airflow parameters (pressure, temperature, and mass flow) through its components, based on given boundary conditions. For the purposes of this paper the generated data correspond to an aircraft flying at $8534.4 \mathrm{~m}(28,000 \mathrm{ft})$ with a speed of $0.78 \mathrm{Mach}$, while the ambient temperature is $232^{\circ} \mathrm{K}$ and the ambient pressure is $33 \mathrm{kPa}$. Virtual sensors measure the air flow parameters at various stations across the ECS, as shown in schematic diagram in Figure 3.

According to Liu et al. ${ }^{21}$ the most frequent ECS faults appear in the Primary or Secondary Heat Exchangers (PHX - SHX), the TCV, and the ram air door actuator an the ACM. Furthermore, as it has been reported by industry experts, the ACM is the most frequently replaced component. For this reason, the diagnostic analysis in this work considers faults in the PHX, SHX, and ACM.

Heat exchangers transfer energy from the bleed to the ram airflow, and their performance depends on the material characteristics. As reported by many operators and researchers, ${ }^{17-19}$ the most frequent fault mode in heat exchangers is accumulations of foreign particles on their walls (fouling). As Wright et al. ${ }^{37}$ report, the most significant contributors to heat exchanger fouling are air pollutants generated by the emissions of ground vehicles. Fouling affects heat exchangers performance by:

- Reducing the component's effectiveness ${ }^{17}$

- Reducing the mass flow rate ${ }^{37}$

- Increasing the pressure drop across the component $^{19}$ 


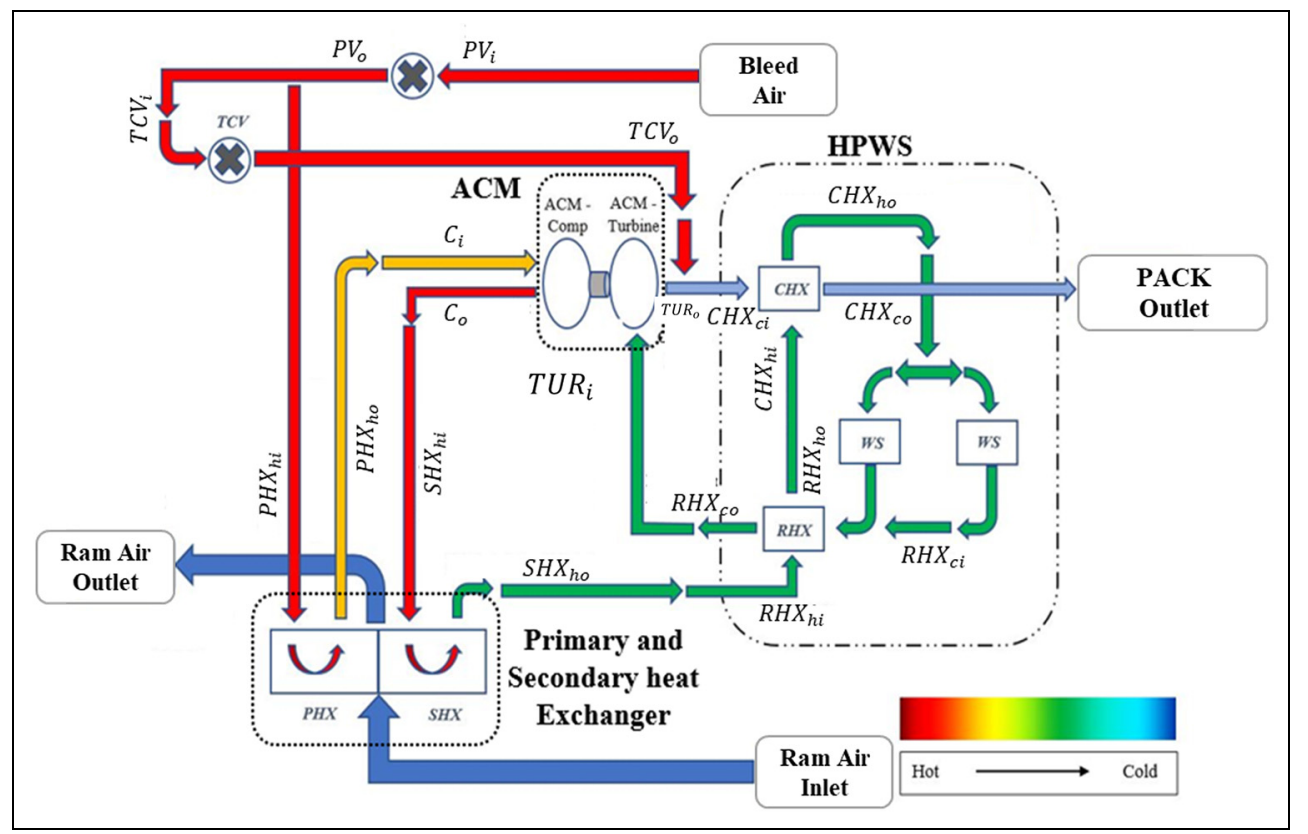

Figure 3. ECS simulation model schematic. ${ }^{20}$

The level to which the component's characteristics are affected depends on the severity of the fault and the type of accumulated foreign particles. The fault simulations conducted in this paper, are based on the hypothesis that fouling affects the component's effectiveness disproportionately to the mass flow rate and the pressure drop characteristics. Therefore, the PHX and SHX faults are emulated by reducing the component effectiveness while keeping the other characteristics unaffected.

As regards the ACM, this is a module that consists of a compressor and a turbine that rotate on a common shaft. However, a fault in either the compressor or turbine results in replacement of the entire ACM module. For the purposes of this work, it is assumed that degradation develops in the turbine. However, ACM turbine fault data are missing from the public domain literature. For this reason, relevant APU turbine fault data will be used for the fault simulation. This is based on the fact that both APU turbine and ACM turbine are essentially the same component, and even though they belong in a different system, it can be reasonably assumed that their fault characteristics are similar. Degradation in the turbine is caused by changes in the geometry of its blades; the degradation effect on this component's characteristics having been discussed in Section 2.1. In the ECS case study, turbine's degradation replicates a fault mode that causes significant reduction in the efficiency compared to the mass flow rate, and for this reason the turbine faults are emulated by decreasing the component's efficiency while the mass flow rate characteristic remains unaffected.

In order to define the maximum simulated level of degradation in the PHX, SHX, and ACM, information from the public domain literature and maintenance manuals were sought. As regards the PHX and SHX degradation levels, information from the aircraft maintenance manuals were leveraged. The aim of this analysis is to diagnose component degradation without having functional system failure. Functional failure exists in cases where the cabin target temperature cannot be achieved, or in-built temperature switches are triggered. More specifically, the Boeing 737-800 ECS has three inbuilt temperature switches that monitor the temperature at the compressor outlet $\left(C O M_{o}\right)$, turbine inlet $\left(T U R_{i}\right)$, and pack outlet (PO). These switches trigger an automatic system shutdown if the temperature in their corresponding locations rises above predefined thresholds, in order to protect the system from catastrophic damage. Also, as mentioned above, in all simulated cases the ECS was able to achieve the cabin target temperature (Pack Outlet - PO).

The maximum degradation limits for these components are defined by running simulations for various degradation severities and ensuring that the thresholds of the inbuilt switches are never exceeded. The initial estimates for the component degradation in the fault simulations are based on the hypothesis that heat exchangers can sustain much higher degradation severity, compared to turbines. Moreover, since fault simulation studies for ACM turbines are missing from the public domain literature, typical degradation levels that correspond to a GT turbine (Section 2.1) are used for the ACM turbine. By taking into account the thresholds of the inbuilt switches, the target temperature and the simulation results, the maximum simulated decrease in the PHX and SHX effectiveness is set up to $50 \%$ and the maximum reduction in the turbine's efficiency at $5 \%$. Figure 4 shows the temperature profile across the ECS for: 


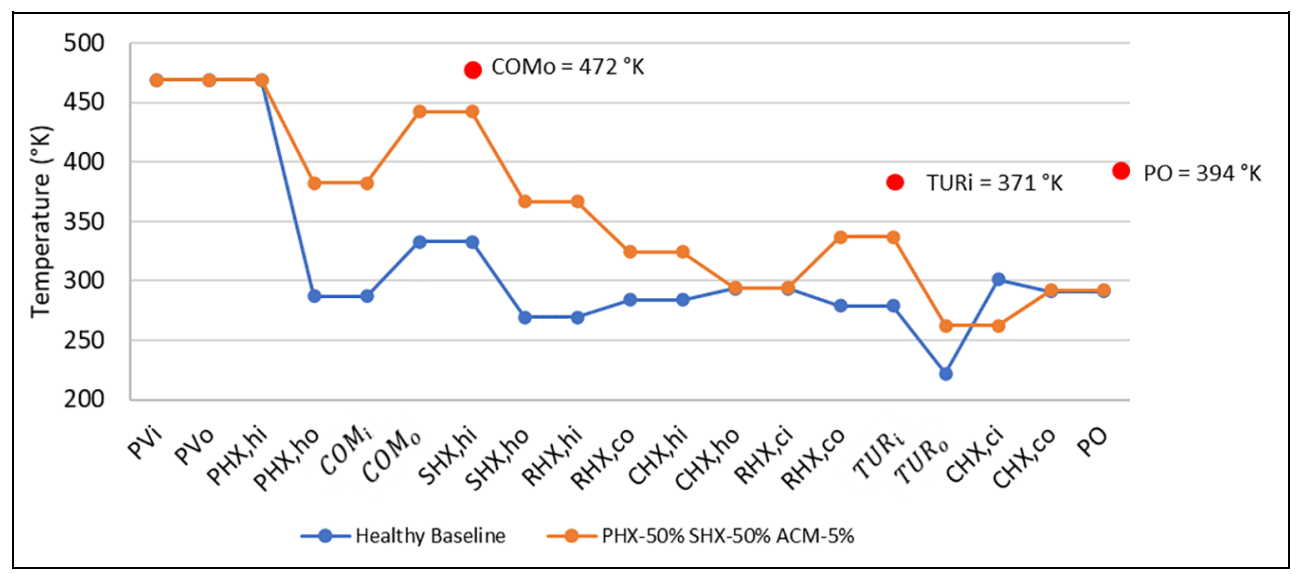

Figure 4. ECS temperature profile comparing the healthy and worst-case fault scenario superimposing the temperature switches.

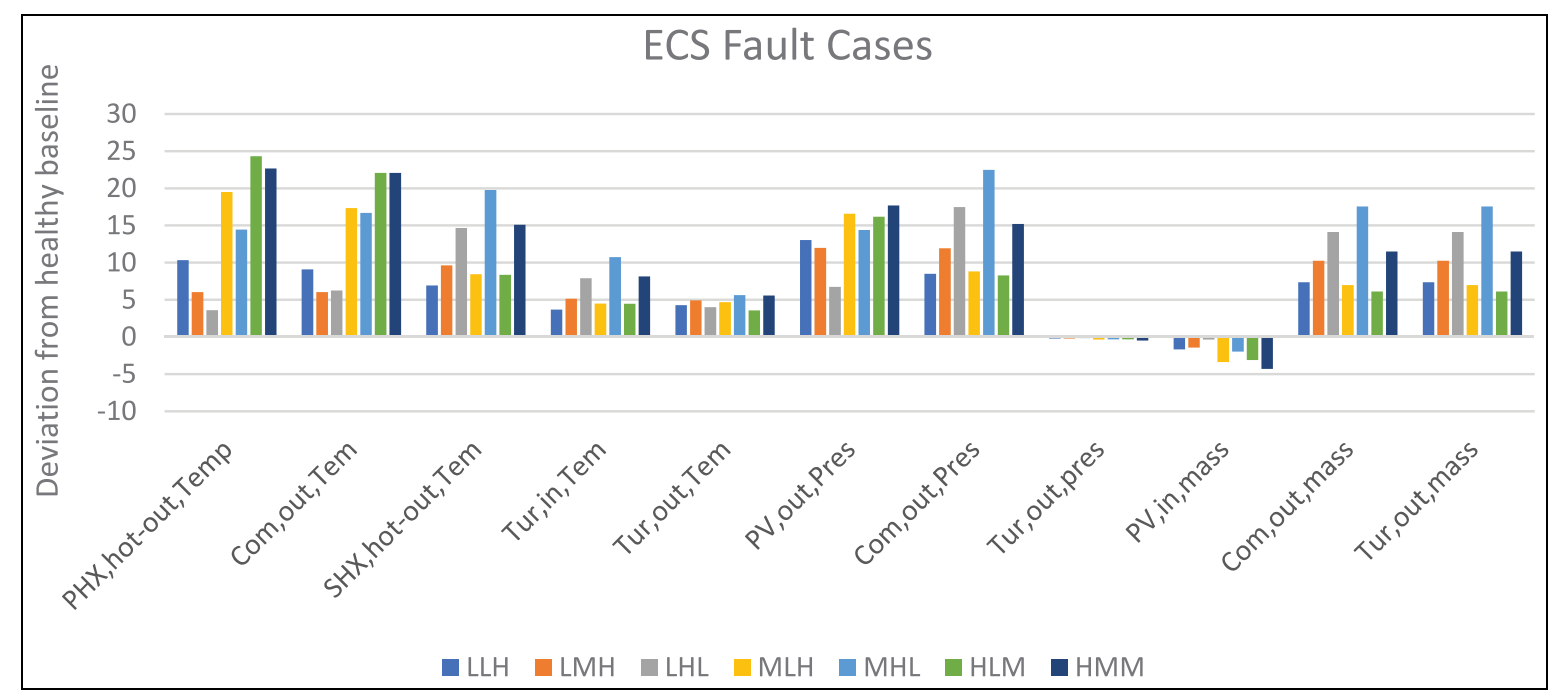

Figure 5. ECS fault combinations.

- the case where all components are healthy.

- the case where all components have the maximum degradation level.

In this figure, even under the highest degradation severity, the thresholds of the temperature switches are not exceeded, and the target temperature (PO) is achieved.

Furthermore, Figure 5 shows the changes imposed on the symptom vector by different fault combinations within the defined boundaries. In this figure, the various degradation levels (Low-L, Medium-M, or High$\mathrm{H}$ ) are imposed on the PHX, SHX, and ACM degradation (in this order). For example, the orange case ( $\mathrm{L} \mathrm{H}$ L) corresponds to a condition where the degradation in the PHX is Low, in the SHX is High and in the ACM is Low. It can be seen that different degradation conditions result in similar changes in the system's performance; thus, the diagnosis of these components is a challenging task.

\section{Connection of the degradation severity regions with maintenance decisions}

The definition of the appropriate thresholds, between the degradation severity classes, is an important issue that heavily affects maintenance action. Their precise identification requires empirical knowledge of the system under examination as well as data that monitors how quickly degradation grows in each component. Major considerations that were taken into account in order to define the thresholds between the different fault severity classes in this work are: 


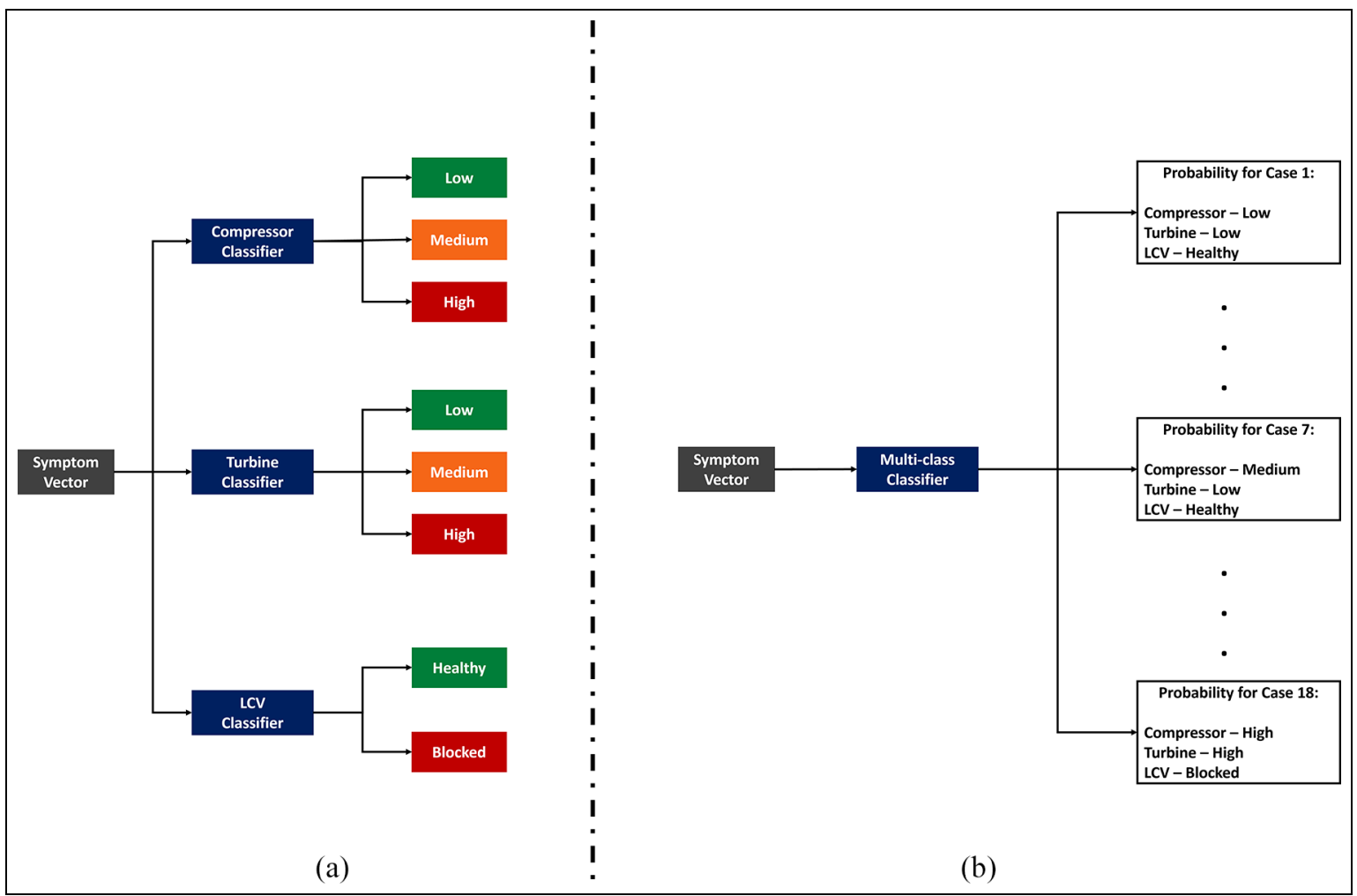

Figure 6. Diagnostic approaches: (a) first diagnostic approaches and (b) second diagnostic approaches.

- Typical levels of degradation (as reported by the public domain literature)

- Engineering judgment that takes into account the maintenance decision that corresponds to each severity region.

Based on the corresponding maintenance actions for each fault severity region, identification of the "Medium" severity class can be considered as the most useful, since it informs the operator that a component should be replaced soon by taking into account the aircraft operational availability and the scheduled maintenance periods. If "Medium" degradation is detected on a component, then the aircraft can continue its operation and the "Medium" degraded component can be replaced when the aircraft is grounded for another scheduled or unscheduled maintenance activity. Thus, this approach optimizes the aircraft's availability by maximizing component useful life, while protecting the system from unexpected faults.

The "High" severity class corresponds to a condition that a component is very close to failure and therefore the operator is advised to perform maintenance immediately. Finally, the "Low" severity class corresponds to a situation under which the system is "almost healthy," and for this reason no maintenance action is advised. Precise identification of the "Low" degradation regions reduces unnecessary component replacement and consequently protects the system from over-maintenance. The necessary data to accurately define the thresholds for the fault severity classes is not known to the authors, and for this reason, the focus of this analysis is to assess the ability of the proposed diagnostic techniques to correctly differentiate between the different fault severity regions. This can, however, be changed by a knowledgeable user who is able to define more realistic thresholds.

\section{APU diagnostic analysis}

\section{Diagnostic framework: Two approaches}

As stated in the beginning of this paper, the target of this analysis is to develop diagnostic techniques that can identify the degradation severity level of the examined components, based on the hypothesis that degradation can develop simultaneously in all components. In this paper, two different diagnostic approaches are compared, and their advantages and limitations are discussed. Under the first approach (Figure 6(a)), for each component under investigation, a classification algorithm is used to identify the corresponding component's health state. In respect to the multiple fault diagnosis framework, the training strategy adopted aims to create classifiers that are able to identify the degradation severity level of their corresponding component by taking into account that the other components can have various degradation levels. For this reason, the training scenarios for each APU and the ECS component follows the generic example in Table 4 . The data used to train "Component 1" classifier are labeled Low, Medium, or High, depending on the degradation level 
Table 4. Generic example of the training scenarios for one component.

Training scenarios for "Component I"

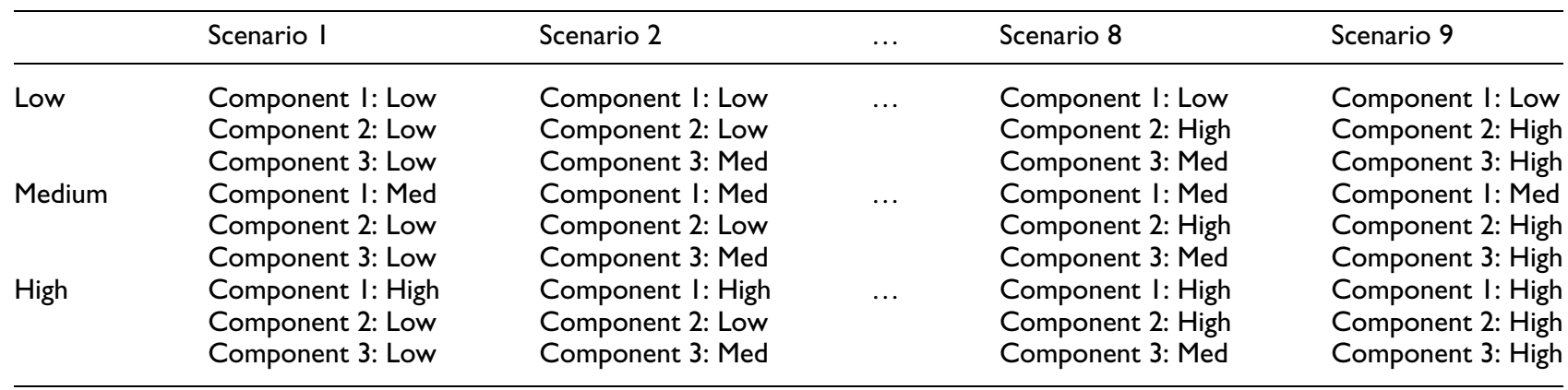

of the examined component (in this e.g. "Component 1 "), while considering that other components are subject to various levels of degradation. In order to achieve this, the data are organized as shown in Table 4. Namely, in each row of Table 4, the degradation level of "Component 1" does not change (Low, Medium, or High), and the nine different scenarios present all possible fault combinations that can be generated while the health state of Components 2 and 3 are subject to Low, Medium, and High degradation. For example, in the "Medium" row, "Component 1" is under medium severity degradation for all nine scenarios, while Components 2 and 3 are subject to different fault combinations. In this way the methodology allows generation of labeled datasets for each component health state, by taking into account the fact that the other system components can be under various degradation levels.

As regards the APU, that is analyzed in this section, six different training scenarios are considered for the compressor and turbine and nine training scenarios are considered for the LCV. Each training scenario, for the compressor and turbine, includes 150 randomly selected cases while each training scenario for the LCV incudes 100 cases. In total each training dataset includes 900 training cases.

The aim of this work is not to design a new classification algorithm that can define the decision boundary more precisely compared to the state-of-the-art. Instead, the ultimate target of this analysis is to evaluate the two diagnostic approaches proposed above, evaluate their effectiveness (correctly diagnosing the system health state), and investigate their applicability to a different system (the ECS). For this reason, in order to select the most appropriate algorithm for each component's classifier, the most commonly used classification algorithms are compared and the algorithm that shows the best performance is selected. This means that, depending on the characteristics of each component training dataset, a different algorithm can be used.

In order to select the most appropriate classifier for each examined component, the MATLAB Classification Learner Toolbox ${ }^{38}$ was leveraged. This tool allows quick training, validation, and testing to the most commonly used classification algorithms. For each examined component, the training dataset was used to train and validate all available algorithms in MATLAB's toolbox and the classifier that showed the best accuracy at the validation stage is selected. The accuracy of each classifier is calculated by using the 10fold cross validation method. Under this validation method, the training dataset is separated in subsets, each subset consisting of 10 training cases. Following that, the algorithm is trained repeatedly, and under each training cycle, a subset that is not considered for training is used to validate the classification results. This process continues until all subsets have been used to validate the trained algorithm. Finally the accuracy is defined by calculating the ratio of correct predictions against all prediction during the validation stage. By following this training methodology, the SVM using a linear kernel function was selected as the compressor classifier (98.4\% accuracy during the validation stage), the SVM using a cubic kernel function was chosen as the turbine classifier (98\% accuracy during the validation stage), and the logistic regression was selected as the LCV classifier (99.8\% accuracy during the validation stage).

In some cases, a single classifier may prove beneficial in a service environment to remove the necessity to maintain more than one algorithm. For this reason, a second diagnostic approach (Figure 6(b)), that consists of a multi-class classifier that assigns a probability score to the 18 possible fault combinations (Table 5), is also tested. Each one of the 18 possible health states includes 50 randomly selected cases (in total 900 training cases). In order to select the most appropriate algorithm, similarly to the first approach, all available multi-class classifiers in Matlab are tested, and the algorithm with the highest accuracy, for each component, is selected. Finally, the SVM classifier was chosen. The probability scores that are assigned to each classification output correspond to the posterior probabilities for each class and are calculated by minimizing the Kullback-Leibler (KL) divergence between the expected and observed probabilities. The KL divergence measures the similarity between prior probability distribution (expected probabilities) and the posteriori 
Table 5. APU fault combinations.

\begin{tabular}{llll}
\hline Case & Compressor & Turbine & LCV \\
\hline 1 & Low & Low & Healthy \\
2 & Low & Low & Blocked \\
3 & Low & Medium & Healthy \\
4 & Low & Medium & Blocked \\
5 & Low & High & Healthy \\
6 & Low & High & Blocked \\
7 & Medium & Low & Healthy \\
8 & Medium & Low & Blocked \\
9 & Medium & Medium & Healthy \\
10 & Medium & Medium & Blocked \\
11 & Medium & High & Healthy \\
12 & Medium & High & Blocked \\
13 & High & Low & Healthy \\
14 & High & Low & Blocked \\
15 & High & Medium & Healthy \\
16 & High & Medium & Blocked \\
17 & High & High & Healthy \\
18 & High & High & Blocked \\
\hline
\end{tabular}

probability distribution (observed probabilities). By minimizing the KL divergence between the expected and observed probability scores of the possible health states, the posterior probabilities for each test case are calculated. The equations used to calculate the KL divergence as well as iterative method used to minimize the KL divergence are provided in the relevant Matlab documentation. $^{39}$
The testing dataset, which is used to test the APU classifiers includes 50 test cases randomly generated, which remain within the component degradation boundaries and are not the same as the training cases. The classification results for both diagnostic approaches are presented via confusion matrices which compare the simulated health state (vertical axis), with the predicted health state (horizontal axis) for each examined component. The elements in the main diagonal (green) represent correct classification, while false positives (cases diagnosed as faulty but actually healthy) and false negatives (cases diagnosed as healthy but actually faulty) are shown in rose and blue, respectively.

\section{View of the training data}

In order to visualize the different fault classes and assess their level of separation, a PCA on the training dataset for both diagnostic approaches are conducted. Figures 7 and 8 present the first three principal components for the first and second diagnostic approach respectively. By observing Figure 7, it can be seen that the different classes have a relatively good separation for all component classifiers. There exist small regions of overlap in the vicinity of the boundaries of the different classes. As will be seen in the rest of this section, the data points within the overlapping regions are the most challenging to diagnose and might result in misclassifications. In

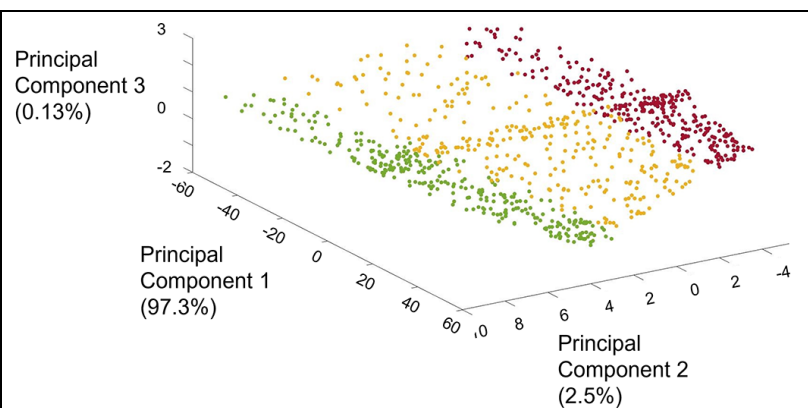

(a)

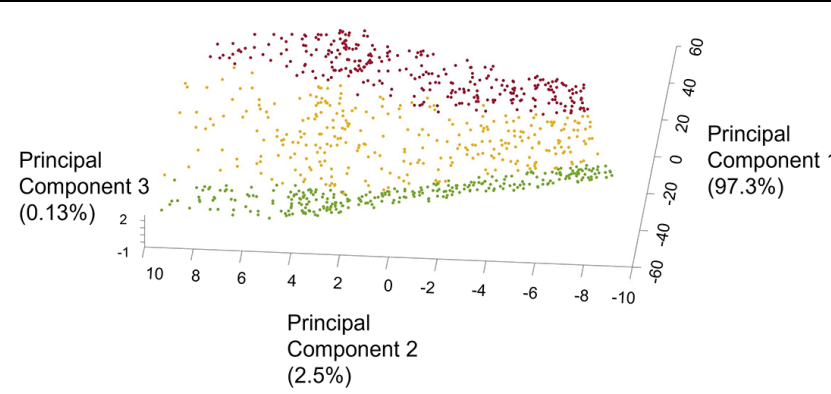

(b)

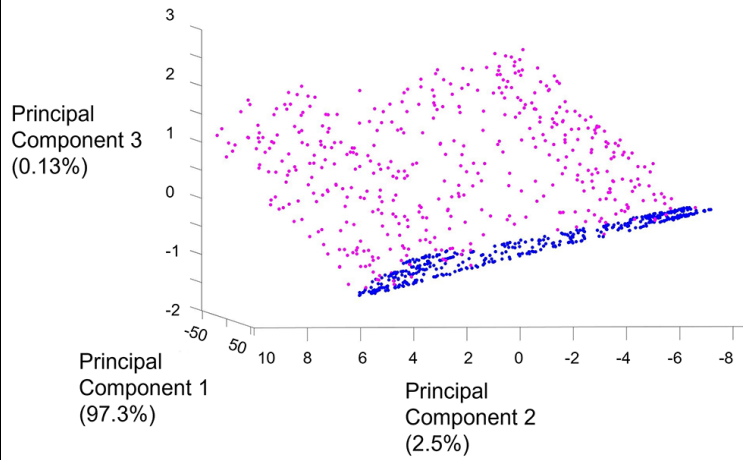

Legend

(c)

Figure 7. PCA for the first diagnostic approach using dense training data: (a) compressor health state, (b) turbine health state, and (c) LCV health state. 


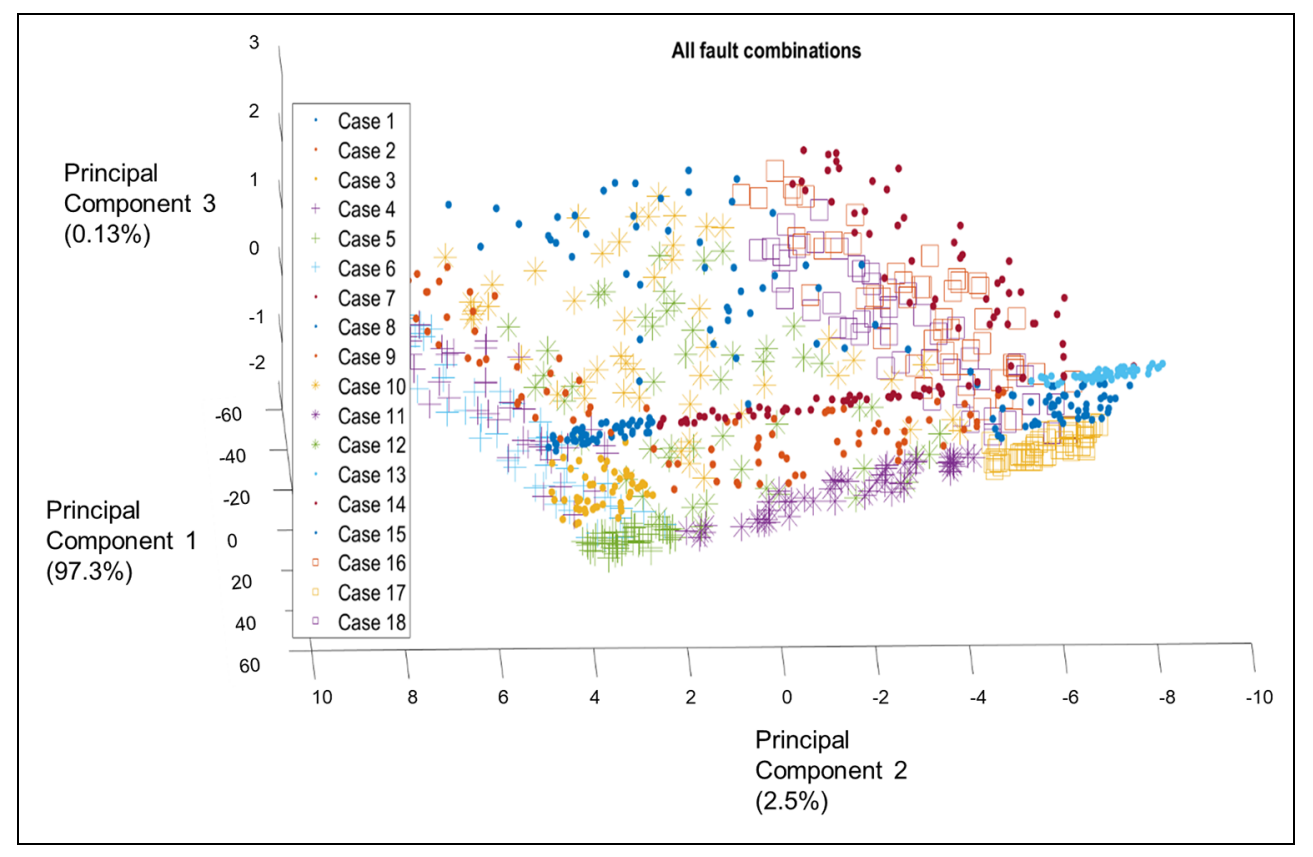

Figure 8. PCA for the second diagnostic approach.

\begin{tabular}{|c|c|c|c|}
\hline & LOW & MEDIUM & HIGH \\
\hline LOW & 7 & 0 & 0 \\
\hline MEDIUM & 0 & 35 & 0 \\
\hline HIGH & 0 & 0 & 8 \\
\hline
\end{tabular}

(a)

\begin{tabular}{|c|c|c|c|}
\hline & LOW & MEDIUM & HIGH \\
\hline LOW & 13 & 0 & 0 \\
\hline MEDIUM & 1 & 29 & 0 \\
\hline HIGH & 0 & 0 & 7 \\
\hline
\end{tabular}

(b)

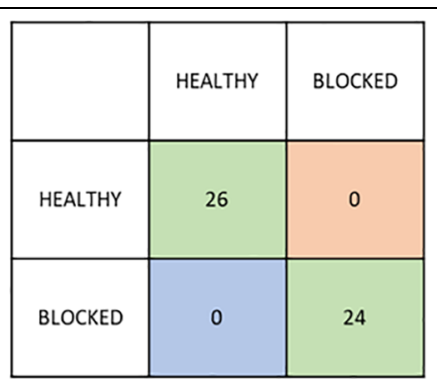

(c)

Figure 9. Diagnostic result of the first approach using test datasets: (a) compressor (SVM), (b) turbine (SVM), and (c) LCV (logistic regression).

the PCA graph for the second diagnostic approach (Figure 8) there exist more overlapping regions, which can be explained by the fact that the same dataset is categorized in more target classes.

As discussed in the rest of this paper, methods that can be adopted to define more accurate decision boundaries are the following:

- simplification of the classification problem by reducing the number of target classes. (This is shown in Section 3.3 in which the results of the first and the second diagnostic approach are compared.)

- training the classification algorithms considering more examples. (In section 5.1 sparser data are used to train the classifiers in the first diagnostic approach.)

\section{Results/analysis}

The results of the first diagnostic approach (Figure 9) show that this technique can correctly predict the component health state for all test cases, apart from one false negative in the turbine classifier. The turbine misclassified case corresponds to a condition in which the degradation level of the compressor is at the upper boundary of the medium severity region, the turbine degradation level is at the lower boundary of the medium severity class and the LCV is blocked $3.7 \%$ (Figure 10). Under these health conditions, the classifier that is responsible to recognize the turbine's health state, erroneously predicted the turbine degradation level "Low," while it is "Medium." This false negative result informs the maintainers that no maintenance action is necessary for the turbine, even though its 


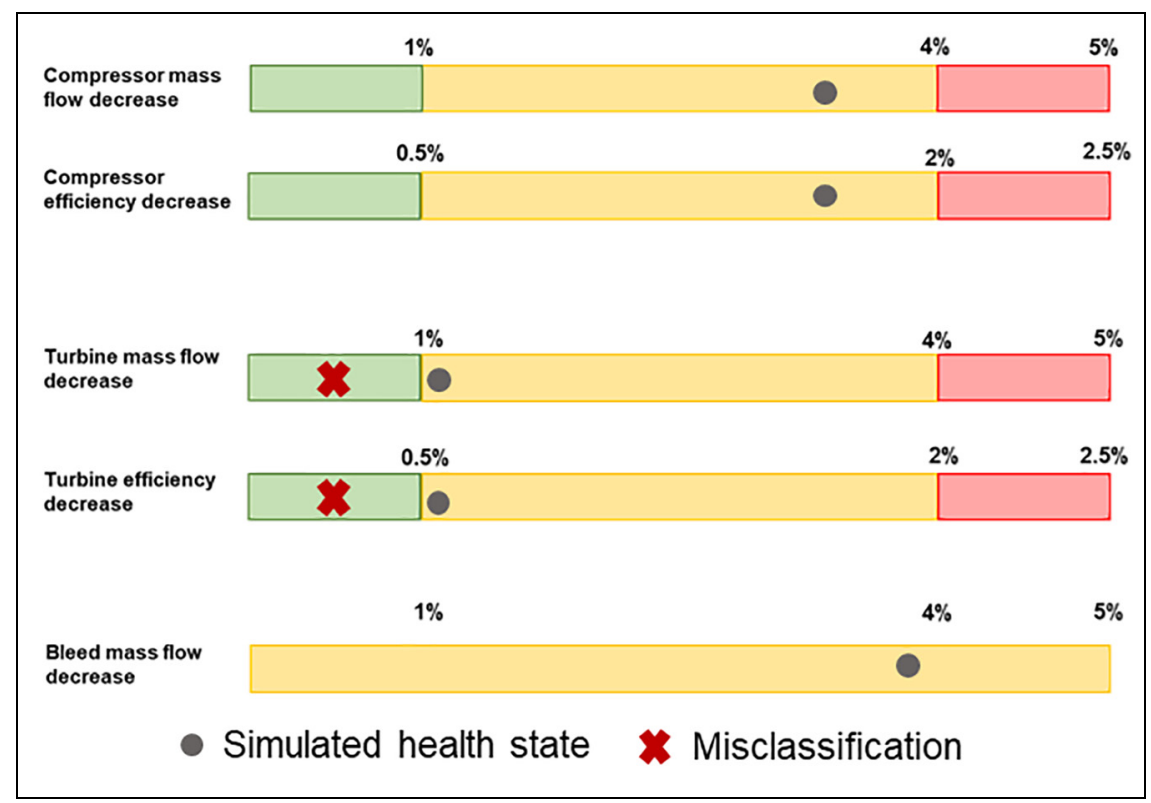

Figure 10. Turbine misclassification under the first diagnostic approach using training datasets.

\begin{tabular}{|c|c|c|c|}
\hline & LOW & MEDIUM & HIGH \\
\hline LOW & 6 & 1 & 0 \\
\hline MEDIUM & 0 & 33 & 2 \\
\hline HIGH & 0 & 1 & 7 \\
\hline
\end{tabular}

(a)

\begin{tabular}{|c|c|c|c|}
\hline & LOW & MEDIUM & HIGH \\
\hline LOW & 11 & 2 & 0 \\
\hline MEDIUM & 1 & 29 & 0 \\
\hline HIGH & 0 & 0 & 7 \\
\hline
\end{tabular}

(b)

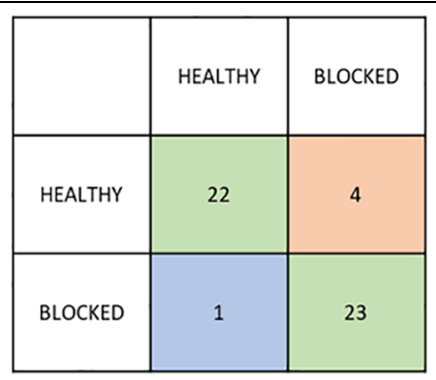

(c)

Figure I I. Diagnostic result of the second approach using test datasets: (a) compressor, (b) turbine, and (c) LCV.

degradation level has increased up to a level that maintenance should be carried out. However, by taking into account the PCA analysis (Figure 7), that showed regions of overlap at the boundaries of the different fault classes, and the fact that different fault cases result in similar fault patterns (Figure 2), the classification results of this diagnostic approach are able to correctly define the relevant decision boundaries and diagnose the component health state quite accurately.

The results of the second diagnostic approach (Figure 11) have more false positives and false negatives (compared to the first approach). The misclassified cases correspond to health conditions that are close to the boundaries of the relevant fault severity classes, similarly to the misclassification observed in the first approach. These results are not unexpected, due to the higher rate of overlapping classes that exist in the corresponding PCA graph (Figure 8). By observing the probability scores of the correctly and erroneously classified test cases, it is seen that the misclassified cases correspond to probability scores below $63 \%$, while the predictions that correspond to a probability score above $63 \%$ are always correct. The fact that each predicted health state is associated with a probability score, can be more helpful as regards the corresponding maintenance decisions. For example, if the diagnostic classifier output corresponds to a high probability score (above 63\%) the relevant maintenance actions can be trusted and carried out without further investigation, however predictions that correspond to low probability scores (below 63\%) would suggest further diagnostic testing.

By comparing the results of the diagnostic approaches described in this section, it can be observed that the first approach is able to predict more accurately the component health state. In the first diagnostic approach, the degradation level of each component is determined by an individual classifier, thus each classifier is required to solve a classification problem that has three (compressor and turbine) or two (LCV) target 


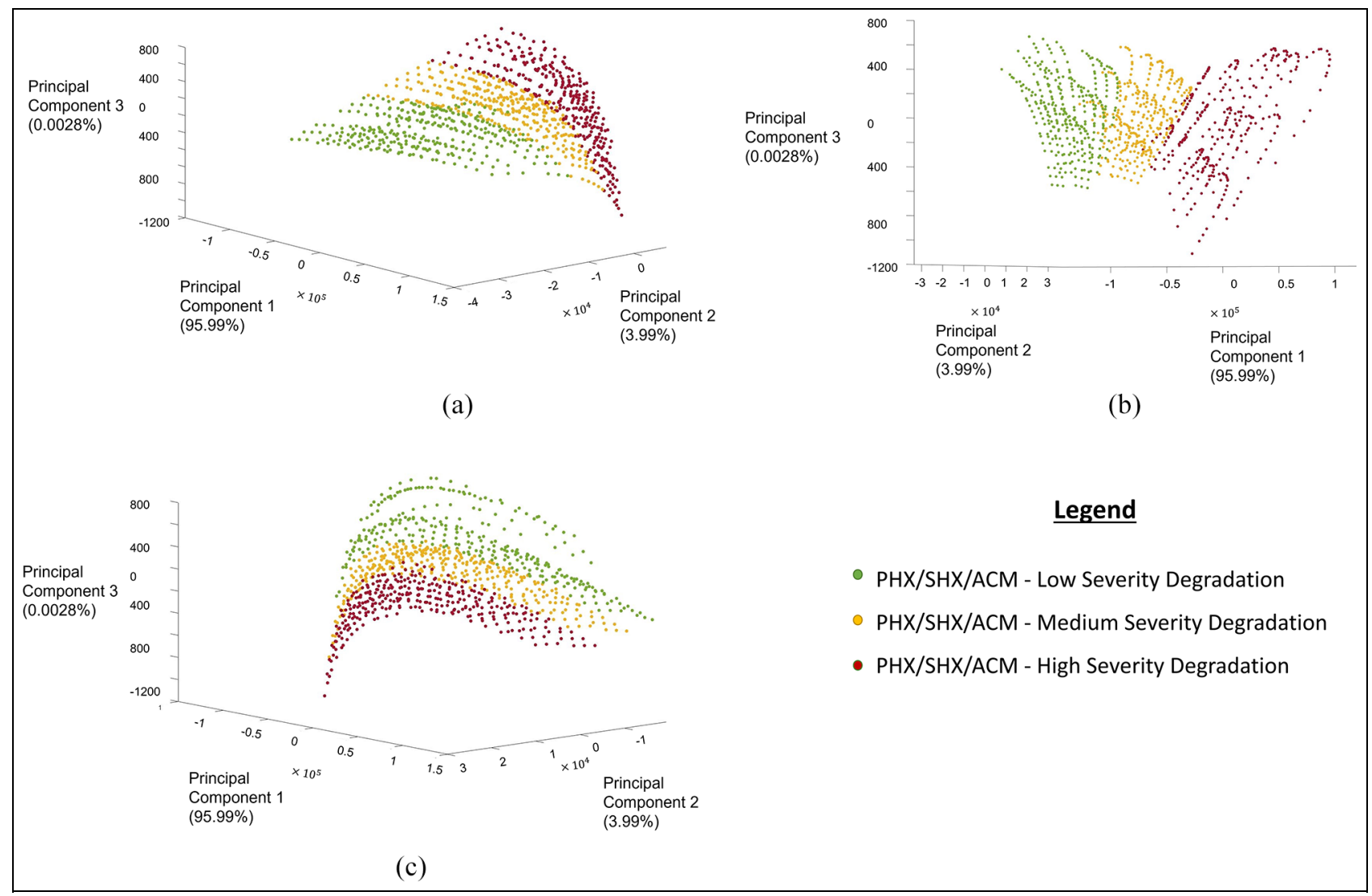

Figure 12. PCA for the training datasets of the ECS components: (a) PHX health state, (b) SHX health state, and (c) ACM health state.

classes. This situation, as seen in the corresponding results, allowed simple classifiers to define accurate decision boundaries for the target classes. In comparison, in the second diagnostic approach the classification output is the solution of an 18-class problem. As observed in the diagnostic results presented above, as the number of classification targets increase, the ability of simple classification algorithms to define correct decision boundaries reduces.

A conclusion from this analysis, is that the accuracy of the diagnostic classifiers depends on the number of the target classes. This means that in order to increase the correct predictions, the relevant classification problem should be simplified, a simplification, the authors suggest, to be based on engineering judgment.

\section{Application of the proposed diagnostic approach to another system: ECS case study}

This section investigates the capability of the diagnostic technique that showed the best performance in the APU case studies (Figure 6(a)), to provide accurate diagnostic predictions when applied on a different system (the ECS). As detailed in Section 2, the ECS components that are considered in this analysis are the PHX, SHX, and ACM (turbine degradation), and their severity levels are presented in Table 3. Similarly, to the APU analysis, training of the ECS component classifiers is based on the training strategy presented in Table 4. For each scenario, there are generated 108 training cases $(108 \times 9=972$ training cases in total for each classifier), and in order to select the classification algorithm for each component, the classifiers in MATLAB classification learner are compared, and the algorithm with the highest accuracy is selected. Finally, for both the PHX and the SHX, an SVM classifier with a cubic kernel function was selected $(99.8 \%$ and $97.9 \%$ accuracy during the validation stage, respectively), and for the ACM, an SVM classifier with a quadratic kernel function was selected $(94.5 \%$ accuracy during the validation stage).

In order to obtain a better insight of the component training datasets, a PCA is conducted for each one of them. The scatter plots of the first three principal components (Figure 12) show good separation among the different fault classes. By considering the separation of the different target classes and the diagnostic results, as in the APU case study, it is expected that the ECS classification algorithms will also be able to correctly predict the component health states.

The datasets used to test the ECS component classifiers were generated independently from the training datasets, while remaining within their corresponding 


\begin{tabular}{|c|c|c|c|}
\hline & LOW & MEDIUM & HIGH \\
\hline LOW & 108 & 0 & 0 \\
\hline MEDIUM & 0 & 72 & 0 \\
\hline HIGH & 0 & 0 & 72 \\
\hline
\end{tabular}

(a)

\begin{tabular}{|c|c|c|c|}
\hline & LOW & MEDIUM & HIGH \\
\hline LOW & 108 & 0 & 0 \\
\hline MEDIUM & 0 & 72 & 0 \\
\hline HIGH & 0 & 0 & 72 \\
\hline
\end{tabular}

(b)

\begin{tabular}{|c|c|c|c|}
\hline & LOW & MEDIUM & HIGH \\
\hline LOW & 72 & 0 & 0 \\
\hline MEDIUM & 0 & 72 & 0 \\
\hline HIGH & 0 & 0 & 108 \\
\hline
\end{tabular}

(c)

Figure 13. Example of test results of ECS components classifiers: (a) PHX (SVM), (b) SHX (SVM), and (c) ACM (SVM).

degradation boundaries. The test results showed that, for all test cases, the component classifiers are able to correctly predict the component's health state. In order to demonstrate the ability of the classification algorithms to correctly differentiate between the component's health states, even in cases where the different fault combinations impose similar changes on the systems performance, the cases presented in Figure 5 are used as a representative example;

- Fault combination 1: PHX - Low, SHX - Low, ACM - High

- Fault combination 2: PHX - Low, SHX - Medium, ACM - High

- Fault combination 3: PHX - Low, SHX - High, ACM - Low

- Fault combination 4: PHX - Medium, SHX - Low, ACM - High

- Fault combination 5: PHX - Medium, SHX High, ACM - Low

- Fault combination 6: PHX - High, SHX - Low, ACM - Medium

- Fault combination 7: PHX - High, SHX Medium, ACM - Medium

For each fault combination there were generated 36 test cases with different severity levels, so in total there are used 252 different test cases. Similarly to the APU case study, the test data for the ECS classifiers was generated independently from the training data whilst remaining within the components fault severity boundaries. The confusion matrices in Figure 13 show that the component classifiers are able to correctly identify the component health states for all test cases.

The fact that the proposed diagnostic approach is able to correctly predict the component health states for two different complex system (the APU and the ECS), shows that it can be essentially applied to any complex system. The ability of this approach to provide accurate predictions relies on the training strategy used, under which the classification algorithms are trained to recognize each component's degradation by taking into account that the other components can have various degradation levels, so, training is conducted on the system-level, rather than on the component-level. Furthermore, as it has been already mentioned in Section 3, by using independent classifiers for each examined component, the classification problem is simplified to a three-class problem, so simple classification algorithms are able to define correct decision boundaries.

By concluding this section, it is highlighted that the data visualization by the 3-D PCA provides confidence to the diagnostic results for both the APU and the ECS. More specifically, the training data visualization by PCA for the APU example, provides clear indication of separation between the severity bands and hence provides confidence in the classifier's response. However, two-dimensional projection of the features for the ECS example shows higher levels of overlap between different bands of severity. At first sight this may seem counterintuitive, as the corresponding classifier still seems to provide diagnostic accuracy to an acceptable level. Despite only providing an additional $0.5 \%$ to the retained variability, when projecting in three-dimensions, separation between severity bands becomes easier to observe. Given that PCA projection is essentially a linear representation of the underlying data-structure it is clear that there is a level of system non-linearity that the data-driven classifier is able to learn from the data and hence why separation between different severity bands is not necessarily easy to observe using linear techniques.

\section{Through life diagnostic approach to maintenance}

The results of the diagnostic analysis for the APU and the ECS have shown that the first diagnostic approach (Figure 6(a)) is able to correctly predict the component health states in both systems. The successful application of this approach on real systems depends on the availability of field data, which are necessary to train the classification algorithms robustly and define the thresholds between the different fault classes. However, in some cases, either because the examined systems do not 


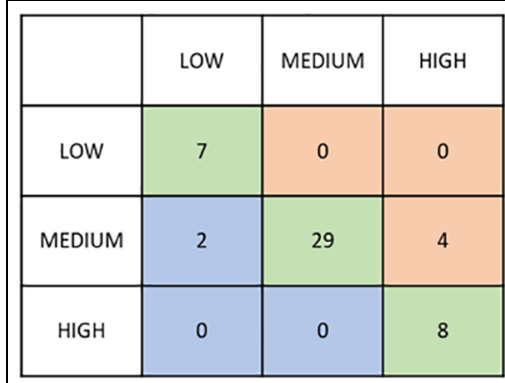

(a)

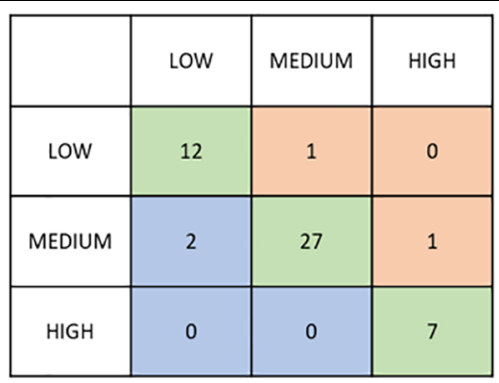

(b)

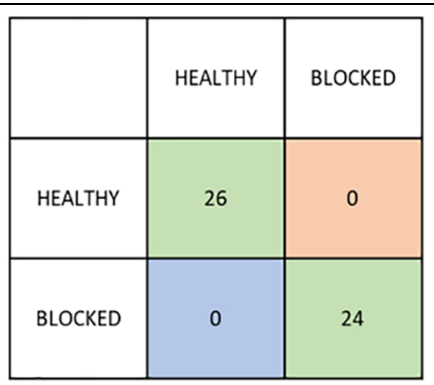

(c)

Figure 14. Diagnostic test result of the first approach (sparse training datasets): (a) compressor (SVM), (b) turbine (SVM), and LCV (logistic regression).

have many in-build sensors (legacy systems), or because new designs are considered, only sparse datasets are available. In order to investigate how severely the diagnostic results are affected by sparse training data, the first part of this section investigates the sensitivity of the proposed diagnostic approach on sparse data. In the second section the findings of the diagnostic analysis are generalized and a Through Life Diagnostic Approach (TLDA) for new and legacy systems is proposed. The overall aim of the TLDA is to contribute toward Condition Based Maintenance (CBM), which is a maintenance strategy that optimizes maintenance plans and results in optimum asset management. ${ }^{40}$

\section{Implementation challenges}

This section aims to discuss challenges that can arise when implementing the discussed diagnostic approach in real systems. A frequent problem that exists in industry, is related with the availability of field data used to train diagnostic algorithms. Therefore, it is necessary to assess the sensitivity of the discussed diagnostic approaches to the density of the training datasets. The analysis in this section aims to investigate how severely the diagnostic results are affected when trained on datasets containing sparse data, and how maintenance decisions are influenced. In order to examine this, the first diagnostic approach (Figure 6(a)) is considered, only for the APU case study. The classification algorithms are trained using data that correspond to the scenarios in Table 4, however, under this analysis, the training cases for each scenario are reduced.

To examine the effect of sparse data each compressor and turbine scenario in Table 4 now includes 30 training cases and each LCV scenario includes 20 training cases. So, each training dataset includes 180 training cases in total. The diagnostic results based on sparse training data are presented in the confusion matrix in Figure 14, and as expected, the predictions based on sparse training datasets deteriorate compared to the predictions of the same diagnostic approach trained on more densely populated datasets (Figure 9). The false positive and false negative results correspond to cases in which the degradation severity of the misclassified components is close to the boundary of the corresponding regions, similar to the example presented in Figure 10. This phenomenon is attributed to the fact that the sparse data used to train the diagnostic classifiers are not able to represent the target classes as well as the training datasets, and consequently the definition of the relevant decision boundaries is less precise.

Nevertheless, it is observed that in most cases, the classifiers are able to correctly predict the component health states, which means that in some cases, the results based on sparse training data can be useful for maintenance decisions. More specifically, the LCV classifier is always able to correctly predict the LCV health state. Thus, the predictions for this component can be always trusted. Furthermore, it is observed that both the compressor and turbine classifiers predict correctly the "High" severity degradation for their respective components. This means that these algorithms can protect the components from a severe degradation and inform the operator for a possible system's fault. However, both the compressor and turbine classifiers might result in unnecessary component replacements or confuse a "Medium" degraded component with a "Low" degraded one. Thus, depending on the risk assessment of each operation, a maintenance decision can be made.

There is an interim solution to the dilemma articulated above, the lack of large field datasets, and it is very much the approach suggested by this work. A healthy simulation can be calibrated from test data (field or experimental) and because it is based on physics, the simulation can be trusted to act rationally when faults are inserted. ${ }^{15}$ These simulations and their accompanying diagnostics serve as the working system as new data arrives from the field. As this new data arrives and is examined the simulations are further validated or can be adjusted to accommodate the new information. In this way the best predictive knowledge is always used. 


\subsection{Through-Life Diagnostic Methodology}

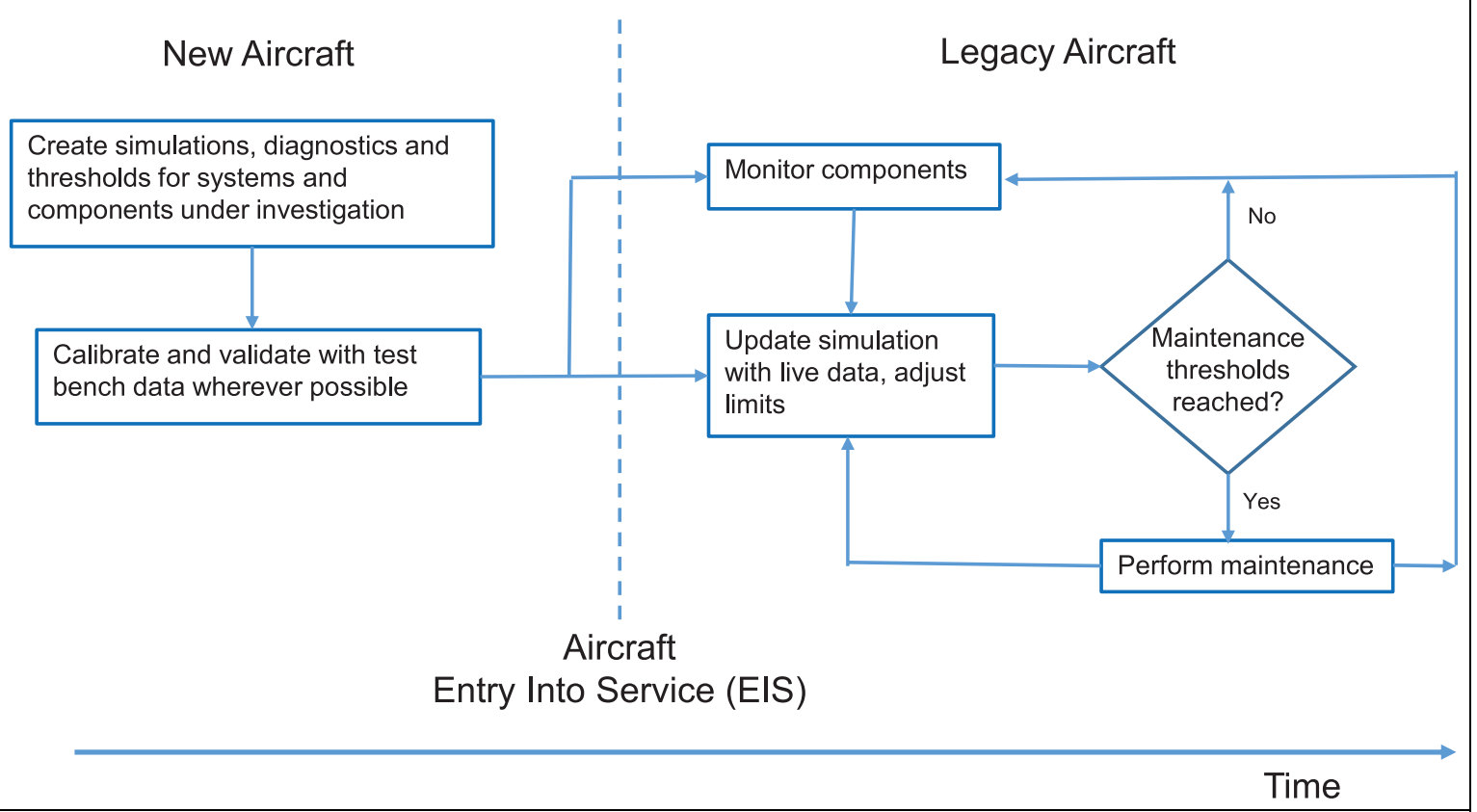

Figure 15. Process for through life maintenance using current diagnostic methodology.

At this point it should be highlighted that the availability of field data discussed above, is not the only issue that might exist when applying the proposed diagnostic methodology on real systems. A number of different implementation challenges might be relevant for other cases, depending on the number of interdependent components, or the characteristics of the examined fault modes (static or dynamic). For example, in some systems due to the fact that many components are strongly interdependent, the application of the proposed approach might result at an unmanageable number of components that should be simultaneously examined. In such cases, a potential solution is the categorization of the components in appropriate subgroups and following that, application of the proposed diagnostic methodology to each subgroup. Also, in cases that require timeseries analysis due to dynamic fault modes (e.g. valve fluctuation), the proposed approach requires modifications in order to take into account the dynamic nature of the fault. A potential approach for dynamic fault detection is to breakdown the timeseries data in multiple partitions, in order to capture different phases of the dynamics, and thereafter, apply the diagnostic methodology as many times as the selected partitions.

Nevertheless, since the architecture of the proposed diagnostic methodology is based on systemindependent features, this method can be potentially applied to many different systems (not only the APU and the ECS) subject to appropriate modifications. To this end, in the next section, an approach to Condition
Based Maintenance considering the diagnostic methodology discussed in this paper is presented.

\section{Through-life diagnostic methodology}

Condition Based Maintenance has been seen as the next paradigm in maintenance action ${ }^{40}$ for a number of years. It involves closely monitoring aircraft components and triggering maintenance depending on their condition, rather than performing scheduled maintenance, which is seen as overly intrusive and expensive to operate. Now that the diagnostic approach has been shown to work on two major aircraft systems, that is, APU and ECS, it is appropriate to consider a methodology that could be used throughout the life of the aircraft in support of CBM.

Figure 15 shows how the current diagnostic approach could be built into a process for maintenance. Starting with a new aircraft, physics-based simulations (like those shown previously for the APU and ECS) can be built from design concepts and data. Component degradation can be built into these models by considering previous aircraft systems and their faults. While this system cannot be totally verified, as no complete aircraft is available for testing at this point, it can be calibrated with component test data and any other available information, such as that from previous aircraft. Note that this option is not possible for a data driven approach.

Upon Entry Into Service (EIS) these simulations and diagnostics, sometimes called digital twins, are the best 
approximation as to system and component life that exist. They can be continuously updated through the aircraft's life by using the monitored data from instrumented components. Note that this is only possible because of the approach used, that is, that all components can degrade, rather than the classical approach of assuming all other components are healthy. Assessing the degradation level against the set maintenance thresholds is continuously performed, with levels being adjusted from engineering judgment. When maintenance is performed the information can be used to adjust the diagnostic limits and monitoring continued.

\section{Concluding remarks}

The overall goal of this work has been to propose a methodology that is able to diagnose faults in the presence of other degraded components, using physicsbased simulation models. The proposed diagnostic methodology was demonstrated on the APU and then applied successfully to the ECS, adding further credence to the method. The success of the proposed diagnostic method relies on the architecture of the diagnostic approach (a dedicated classifier for each examined component) and the training strategy (multiple scenarios considering multiple component faults simultaneously). Moreover, the confidence in the diagnostic results is also supported by the fact that the data visualization by 3-D PCA shows clear separation between the target classes. The classifiers were able to identify very accurately the non-linearities in the training data and could define correct decision boundaries. The diagnostic framework allows a "traffic light" system (green, amber, red) for maintenance to be developed. The association between the component degradation severity and a potential maintenance activity was outlined and discussed.

Also, in Section 5.1 there is discussed a typical implementation challenge of the proposed methodology and the corresponding analysis concluded that the approach does not work with sparse data, a shortcoming that can be remedied with simulated data. Finally it is mentioned that since each case study might encapsulate different implementation challenges, careful evaluation of the specific characteristics of the examined system should be conducted and appropriate modification to the diagnostic methodology might be necessary to achieve successful diagnostics.

Furthermore, based on the findings of the diagnostic analysis on the APU and ECS, a through life CBM maintenance paradigm, applicable to new or legacy designs, is proposed. The application of the CBM approach can lead in optimized maintenance strategies and consequently increased asset availability.

Finally, in order to enhance the proposed approach to CBM, aspects that will be explored in future work is the enhancement the proposed diagnostic methodology by considering more implementation challenges (e.g. how a large number of components can be managed, and how the methodology can be applied under dynamic faults). Also, further analysis can be carried out on issues related to sensors uncertainty. This can define the sensor characteristics that should be installed on the systems in order to allow accurate fault detection. Finally, another interesting challenge is the identification of the minimum number of sensors that are required to detect a predefined number of fault modes.

\section{Acknowledgements}

The authors would like to thank Boeing for their support of this project.

\section{Declaration of conflicting interests}

The author(s) declared no potential conflicts of interest with respect to the research, authorship, and/or publication of this article.

\section{Funding}

The author(s) disclosed receipt of the following financial support for the research, authorship, and/or publication of this article: This work has been funded by The Boeing Company as part of their collaboration with Cranfield University's IVHM Centre.

\section{ORCID iDs}

Christos Skliros (D) https://orcid.org/0000-0001-94069655

Steve King (iD https://orcid.org/0000-0001-5949-4744

\section{References}

1. Saxon $\mathrm{S}$ and Weber M. A better approach to airline costs. In: McKinsey \& company travel, transport \& logistics. McKinsey \& Company, https://www.mckinsey. com/industries/travel-transport-and-logistics/our-insigh ts/a-better-approach-to-airline-costs (2017, accessed 10 September 2020).

2. International Air Transport Association. Preliminary analysis of MCTF FY2017 data. In: 14th maintenance cost conference, 2018, https://www.iata.org/contentassets/ f03b1a4b79534b99802f10cd23b19ec2/1000-1030-prelimanalysis-of-mctf-data-iata.pdf

3. Jennions IK. (ed.). Integrated vehicle health management - perspectives on an emerging field, Warrendale, PA: SAE International, 2011.

4. Yabsley A and Ibrahim Y. Study on maintenance contribution to life cycle costs: aircraft auxiliary power unit example. In: Proceedings of the IEEE international conference on industrial technology, Chengdu, China, 2008. New York: IEEE. DOI: 10.1109/ICIT.2008.4608331.

5. Mofokeng T, Mativenga PT and Marnewick A. Analysis of aircraft maintenance processes and cost. Procedia CIRP 2020; 90: 467-472.

6. Skliros C, Ali F and Jennions I. Experimental investigation and simulation of a Boeing 747 auxiliary power unit. J Eng Gas Turbine Power 2020; 142(8): 081005. 
7. Li YG. Performance-analysis-based gas turbine diagnostics: a review. Proc IMechE, Part A: J Power and Energy 2002; 216(5): 363-377.

8. Tahan M, Tsoutsanis E, Muhammad M, et al. Performance-based health monitoring, diagnostics and prognostics for condition-based maintenance of gas turbines: a review. Appl Energy 2017; 198: 122-144.

9. Bettocchi R, Pinelli M, Spina PR, et al. Artificial intelligence for the diagnostics of gas turbines - part 1: neural network approach. J Eng Gas Turbine Power 2007; 129(3): 711-719.

10. Bettocchi R, Pinelli M, Spina PR, et al. Artificial intelligence for the diagnostics of gas turbines - part II: neurofuzzy approach. J Eng Gas Turbine Power 2007; 129(3): 720-729.

11. Gorinevsky D, Dittmar K, Mylaraswamy D, et al. Model-based diagnostics for an aircraft auxiliary power unit. In: IEEE international conference on control applications, Glasgow, Scotland, UK, 2002.

12. Vianna WOL, Gomes JPP, Galvão RKH, et al. Health monitoring of an auxiliary power unit using a classification tree. In: PHM 2011 proceedings of the annual conference prognostics and health management society, Montreal, QC, 2014, pp.293-299.

13. Pascoal RM, Vianna WO, Gomes JP, et al. Estimation of APU failure parameters employing linear regression and neural networks. In: PHM 2013 proceedings of the annual conference prognostics and health management society, New Orleans, LA, 2013, pp.664-670.

14. Guralnik V, Mylaraswamy D and Voges H. On handling dependent evidence and multiple faults in knowledge fusion for engine health management. In: 2006 IEEE aerospace conference, Big Sky, MT, 2006. New York: IEEE. DOI: 10.1109/AERO.2006.1656100.

15. Skliros C, Ali F and Jennions I. Fault simulations and diagnostics for a Boeing 747 auxiliary power unit. Expert Syst Appl 2021; 184: 115504. DOI: 10.1016/ j.eswa.2021.115504.

16. Jennions I, Ali F, Miguez ME, et al. Simulation of an aircraft environmental control system. Appl Therm Eng 2020; 172: 114925.

17. Najjar N, Gupta S, Hare J, et al. Optimal sensor selection and fusion for heat exchanger fouling diagnosis in aerospace systems. IEEE Sens $J$ 2016; 16(12): 4866-4881.

18. Shang $\mathrm{L}$ and Liu G. Heat exchanger fouling detection in a simulated aircraft engine bleed air temperature control system. In: IEEE/ASME international conference on advanced intelligent mechatronics, AIM, Montreal, QC, 2010, pp.774-778. DOI: 10.1109/AIM.2010.5695895.

19. Shah S, Liu G and Greatrix DR. On-line fouling detection of aircraft environmental control system cross flow heat exchanger. In: 2009 IEEE international conference on mechatronics automation ICMA 2009, Changchun, China 2009, pp.2940-2945. DOI: 10.1109/ICMA.2009.5246062.

20. Jennions I and Ali F. (April 2, 2021). "Assessment of Heat Exchanger Degradation in a Boeing 737-800 Environmental Control System." ASME. J. Thermal Sci. Eng. Appl. December 2021; 13(6): 061015. https://doi.org/ $10.1115 / 1.4050324$
21. Liu C, Sun J, Wang F, et al. Bayesian network method for fault diagnosis of civil aircraft environment control system. Proc IMechE, Part I: J Systems and Control Engineering 2020; 234(5): 662-674.

22. Hare J, Gupta S, Najjar N, et al. System-level fault diagnosis with application to the environmental control system of an aircraft. SAE technical paper 2015-01-2583, 2015. DOI: $10.4271 / 2015-01-2583$.

23. Sadough Vanini ZN, Meskin N and Khorasani K. Multiple-model sensor and components fault diagnosis in gas turbine engines using autoassociative neural networks. $J$ Eng Gas Turbine Power 2014; 136(9): 091603.

24. Joly RB, Ogaji SO, Singh R, et al. Gas-turbine diagnostics using artificial neural-networks for a high bypass ratio military turbofan engine. Appl Energy 2004; 78(4): 397-418.

25. Lee YK, Mavris DN, Volovoi VV, et al. A fault diagnosis method for industrial gas turbines using Bayesian data analysis. J Eng Gas Turbine Power 2010; 132(4): $1-6$.

26. Sampath $\mathrm{S}$ and Singh R. An integrated fault diagnostics model using genetic algorithm and neural networks. $J$ Eng Gas Turbine Power 2006; 128(1): 49-56.

27. Palmer KA, Hale WT, Han L, et al. Built-in test design for fault detection and isolation in an aircraft environmental control system. IFAC-PapersOnLine 2016; 49(7): $7-12$.

28. Lin Y, Zakwan S and Jennions I. A Bayesian approach to fault identification in the presence of multi-component degradation. Int. J Progn Heal Manag 2017; 8: 1-9

29. Kurz R and Brun K. Degradation in gas turbine systems. J Eng Gas Turbine Power 2001; 123(1): 70-77.

30. Graf MB, Wong TS, Greitzer EM, et al. Effects of nonaxisymmetric tip clearance on axial compressor performance and stability. Proc. ASME Turbo Expo 1998; 120(4): 648-661.

31. Igie U, Pilidis $P$, Fouflias D, et al. Industrial gas turbine performance: compressor fouling and on-line washing. $J$ Turbomach 2014; 136(10): 101001.

32. Zwebek AI and Pilidis P. Degradation effects on combined cycle power plant performance - part III: gas and steam turbine component degradation effects. J Eng Gas Turbine Power 2004; 126(2): 306-315.

33. Boyle RJ. Prediction of surface roughness and incidence effects on turbine performance. $J$ Turbomach 1994; 116: 745-751.

34. Kurz R, Brun K and Wollie M. Degradation effects on industrial gas turbines. J Eng Gas Turbine Power 2009; 131(6): 062401 (7 pages). DOI: 10.1115/1.3097135.

35. Shang L and Liu G. Sensor and actuator fault detection and isolation for a high performance aircraft engine bleed air temperature control system. IEEE Trans Control Syst Technol 2011; 19(5): 1260-1268.

36. Daigle MJ and Goebel K. A model-based prognostics approach applied to pneumatic valves. Int J Progn Heal Manag 2011; 2(2): 1-16.

37. Wright SJ, Dixon-Hardy DW and Heggs PJ. Aircraft air conditioning heat exchangers and atmospheric fouling. Therm Sci Eng Prog 2018; 7: 184-202. 
38. Mathworks. Train models to classify data using supervised machine learning, https://uk.mathworks.com/help/ stats/classificationlearner-app.html (2015 accessed 11 November 2020).

39. Mathworks. Classify observations using multiclass errorcorrecting output codes (ECOC) model, https://uk. mathworks.com/help/stats/classificationecoc.predict.html \#mw_7566e162-d07a-4719-b1b0-a381c2b78a85 (accessed 11 November 2020).

40. Jennions IK (ed.). Integrated vehicle health management: perspectives on an emerging field. Warrendale: SAE International, 2012.

\section{Appendix}

Notations

\section{Abbreviations}

$\mathrm{ACM}$

Air cycle machine

APU

Auxiliary power unit

CBM

CHX

$\mathrm{COM}$

D-S

ECS

EGT

EIS

EKF

ETC

FMV

GT

HPWS

IATA

LCV

MRO

PHX

PV
RHX Regenerative heat exchanger

SHX Secondary heat exchanger

SVM Support vector machine

TLDA Through life diagnostic approach

TCV Temperature control valve

TUR Turbine

\section{Latin symbols}

$\begin{array}{ll}f & \text { Frequency } \\ \dot{m} & \text { Mass flow } \\ N & \text { Rotational speed } \\ p & \text { Pressure } \\ S & \text { ETC signal } \\ T & \text { Temperature } \\ V & \text { Voltage }\end{array}$

\section{Greek symbols}

$\Delta$

Difference

\section{Subscripts}

bl Bleed

ci Cold inlet

co Cold outlet

com Compressor

$f \quad$ Fuel

hi Hot inlet

ho Hot outlet

I Inlet

in Inlet

o Outlet

out Output

tur Turbine 
$2021-10-26$

\section{Aircraft system-level diagnosis with emphasis on maintenance decisions}

Skliros, Christos

SAGE

Skliros C, Ali F, King S, Jennions I. (2021) Aircraft system-level diagnosis with emphasis on maintenance decisions. Proceedings of the Institution of Mechanical Engineers, Part O: Journal of Risk and Reliability, Volume 236, Issue 6, December 2022, pp. 1057-1077

https://doi.org/10.1177/1748006X211054938

Downloaded from Cranfield Library Services E-Repository 